\title{
Robust nonsingular sliding mode control of the maglev train system: case study
}

\author{
Kammogne Soup Tewa Alain ${ }^{1}\left[\right.$ D $\cdot$ Kenmogne Fabien ${ }^{2} \cdot$ Siewe Siewe Martin $^{3} \cdot$ Fotsin Hilaire Bertrand $^{1}$
}

Received: 10 October 2020 / Accepted: 4 February 2021 / Published online: 22 February 2021

(C) The Author(s) 2021 OPEN

\begin{abstract}
This paper deals with a new approach to explore the precise dynamic response of the maglev system train and its control. Magnetic-suspension systems are characterized by high nonlinearity and open-loop instability which are the core components of maglev vehicles. Firstly, we use the electromagnetics and mechanics laws to derive the mathematical expressions of the proposed maglev system. Analytical investigation and theoretical calculation show that for the specific values of the control system parameters, the maglev system train can be significantly improved. It points out that the inherent nonlinearity, the inner coupling, misalignments between the sensors and actuators, and external disturbances are the main issues that should be considered for maglev engineering. Secondly, a control strategy based on the precise model of a nonsing ular robust sliding mode control is designed to reduce the upper bound of both the uncertainty and interference of the sliding mode controller. This approach presents an added value compared to the new sliding control methods in terms of overshoot and speed of convergence which is designed to control the vertical position of the proposed system. By using rigorous mathematical transformation associated with the adaptation laws in the frequency domain, a sufficient condition is drawn for the stability of the dynamical error based on the Lyapunov theory. This allows us a great possibility for interpreting the operation of the maglev train system. Numerical results are presented to show the effectiveness of our proposed control scheme.
\end{abstract}

Keywords Maglev system · Dynamical analysis · Dynamic model · Sliding mode control · Adaptive control

\section{Introduction}

Maglev systems attract great interest from engineers over the world due to their basic characteristics (contact-less property between the rail and the train, minimal maintenance cost, faster speed and so on) and their different applications such as high-speed, frictionless bearing and levitation of metal slabs during manufacturing [1]. It has a broad spectrum of applications in different domains in day-to-day life for example in transportation $[2,3]$, bearing-less motors [4, 5], bio-medical [6], industries [7, 8], launching of rockets $[9,10]$, in levitation of metals etc. A step-by-step design of an efficient suspension system is globally determined by an in-depth understanding of the train dynamics. It is also essential for the development of the control laws for the effective operation of the Maglev system. In order to be commercially viable, the Maglev train must have certain technical requirements to fully satisfy passengers. Therefore, it is essential to consider the dynamic as well as static stability as an integral part of Maglev design even for the control development.

$\triangle$ Kammogne Soup Tewa Alain, kouaneteoua@yahoo.fr; Kenmogne Fabien, kenfabien@yahoo.fr; Siewe Siewe Martin, martinsiewesiewe@yahoo.fr | ${ }^{1}$ Laboratory of Condensed Matter, Electronics and Signal Processing (LAMACETS), Department of Physic, Faculty of Sciences, University of Dschang, P.O. Box 67, Dschang, Cameroon. ${ }^{2}$ Department of Civil Engineering, Higher Technical Teachers Training College, University of Douala, Douala, Cameroon. ${ }^{3}$ Laboratory of Mechanics, Materials and Structures, Faculty of Science, Department of Physics, University of Yaounde 1, P.O. Box 812, Yaounde, Cameroon. 
From a viewpoint of the mechanics, the dynamics of the mechanical parts are inescapably affected by the electromagnetic properties. A ferromagnetic material suspended to an air spring, which is subject to the electromagnetic force created by the field of an electromagnet. The electrical current flowing along the magnetic circuit is a nonlinear function of the electromagnetic force. consequently, all motions induced in the ferromagnetic material are nonlinear. As a result, extensive dynamical researches have been carried out to achieve satisfactory performance specifications $[11,12]$. Most of these works essentially provide the theoretical basis for the development of the electromechanical systems and then give us great inspiration to further improve the Maglev system. Some approaches for the analysis of suspension systems were strongly associated with the vibration phenomena such as hopf bifurcation, limit cycle and so on. Recently, Yougang et al., investigated the modeling and the control of the low-speed maglev using fuzzy sliding-mode technique [13]. In their work, they used the Euler-Lagrange strategy to construct the nonlinear dynamic model. In the qualitative analysis viewpoint, Lee investigated the occurrence of the Hopf bifurcation and the impact of guide-way parameters on the Maglev system behavior [14]. Following the same idea, Haiyan and collaborators presented an appropriate levitation chassis analysis with a relevant robust position control design of the suspension system under nonlinear periodic disturbance [15]. They demonstrated that the stable suspension of the maglev vehicle is achieved by controlling individual EMS modules. More recently, Zhai and coworkers proposed a calculation and evaluation of the load performance of Maglev in medium-low speed [16]. They provided a load performance of the magnetic levitation system under different load using theoretical calculations associated with the finite element. We note that the typical modeling of the maglev system met the unceasing improvement and is more confronted to the basic requirements of engineering applications, but now-a-days, there are still many technical difficulties, especially for the analysis and design of a levitation system. Despite the numerous works on this topic, there is no relevant study that provides a systematic design procedure in the simpler manner of the Maglev system to the best of the author's knowledge. We notice that the improvement of the Maglev train dynamic is of paramount importance and can be achieved through the perfect knowledge of the behavior of the parameters.

It is worthwhile to recall that, the maglev system suffers from various control complexities such as inherent instability, one direction force input, nonlinearity modeling and exogenous disturbances $[17,18]$. Hence, an external controller is necessary to stabilize the overall system. Abundant control approaches of the maglev systems are based on the linear model which use the linear controller
[19-21] and the references therein. However, this design model tracking performance deteriorates rapidly as deviation with respect to nominal operating point increases. Some modeling technics were proposed by researchers in order to linearize existing models or to develop a theory around the suppression of vibrations in the system. Most researches in literature deal with appropriate nonlinear controllers that control globally instead of locally the dynamic of the system in a real-time. The principal criterion to select the nonlinear controller is strongly based on its ability to give fast and efficient dynamic response i.e. lesser rise time, fast settling time, less peak value, minimum overshoot/undershoot and negligible steady-state error [22]. In addition, the nonlinear controller is designed to deal with the inherent perturbations on the system dynamic. The majority of the nonlinear control presents poor performance and is less efficient against the disturbances. Therefore, the major motivation behind this work is to design an appropriate nonlinear controller for stabilizing the maglev system with best dynamic and also to allow the guide magnets to keep the rail on track by overcoming the effect of disturbances in an efficient way.

Many contributions have been devoted to the development of control strategies of the maglev train. Yang et al., demonstrated that the magnetic levitation subjected to the uncertainty can be efficiently controlled via a particular dynamic surface proposed a robust position control of a magnetic levitation system via dynamic surface control technique of magnetic levitation system containing parameter uncertainty [23]. The position control strategy is constructed to maintain stable perturbed Maglev system. On the other hand, a robust estimator is designed to estimate the system uncertainties using a DSP-based sliding-mode control for an electromagneticlevitation system by Lee et al., [24]. These techniques present the advantages to be simple and consequently easy to be implemented, but they consider only a particular scenario, which may be conservative. Wiboonjaroen and Sujitjorn [25] exploited the linearized version of the Maglev model to derive a novel state-PID feedback controller. We notice that the results obtained penalize the performance of the controller due to the fact that the linear systems do not actually exist. The linearization of such systems inevitably leads to the loss of some important properties of the original systems. Recently, Bobtsov et al., proposed a state observer for sensorless control of maglev systems [26]. More recently, Sun and coworkers developed a minimum levitation unit via the RBF Neural Network for the Maglev systems [27]. The neural network enabled us to improve significantly the proposed strategy as regards time-delay. Although, the promising results obtained by these authors, it appears boring and bulking in the practical case. In [28], a nonlinear control 
method for the electromagnetic suspension system of the maglev train was designed by Junqi and Yuan. They prove that the adjustment of time delay parameters plays a guiding role in avoiding vibration. The non-adaptive time-delayed feedbacks controllers are designed to control the overall system which stands as a drawback since the feedback gains cannot be infinite. On the same way, You-gang Sun et al., presented a robust levitation control of maglev vehicles subject to time delay and disturbances: design and hardware experimentation [29]. Their works show an adequate design of controller based on the Riccati method and sliding-mode technology that can handle efficiency the time delays and disturbances in the control scheme. The basic requirement is the enhancement in the dynamic response of the system so that the proposed controller should have characteristics like: minimal overshoot/undershoot, faster convergence rate, minimal or zero steady state error and reduced chattering and robustness against external disturbance. To satisfy these requirements we choose the nonsingular sliding mode for designing our proposed controller.

Amongst huge control approaches, the application of sliding mode technic to control Maglev system are numerous [30,31] and give many advantages such as fast response, insensitivity to the matching external disturbances, robustness against uncertainties and good transient performance $[32,33]$. It's important to recall that sliding mode structure control has achieved success in various domains. However, from the literature viewpoint, there are only a few works that take into account these procedures magnetic suspension systems area. It is worth to recall that in physical Maglev systems, some significant constraints exist, such as unavailability of all the state variables, matched and/or unmatched uncertainties due to parameter variation, model simplification and/or external disturbances [33-35]. Due to these considerations, it is become a titanic task to design an appropriate controller with the specifics performance. An attempt to overcome perturbations in the system is to use the nonsingular sliding mode control which uses a single control inputs and allows the finite-time stabilization using without requiring the information of the uncertainties [36, 37]. In addition, the singularity and the chattering are eliminated. Nevertheless, this topology of the controller has some weaknesses such as asymptotic stability with a slow response.

The scope of this paper falls into the electro-magnetic suspension technology. We consider a novel dynamical approach associated with a control problem of the nonlinear Maglev system. Facing the challenges previously established, the main contributions of this paper are three-fold:

1. Design and analyze the Maglev system in terms of laws and including stability theories,
2. Propose a robust control strategy for the Maglev train which can ensure the system to be uniformly bounded.

3. Propose an adaptive law to regulate the total system uncertainty, which was not available earlier.

The designing of the novel adaptive robust nonsingular sliding mode control reveals an important contribution of this paper. To the best of the author's knowledge, not of all these aspects of the Maglev have been simultaneously considered in the literature and then become challenging. In this study, we emphasize the need for improvement of Maglev system in their technical performance and the designing of the efficient control strategy which deserves recognition as a profound contribution.

The rest of the paper is organized as follows. Section 2 gives the problem description and dynamical analysis of the maglev system with some associated simulations. Section 3 deals with the designing of the nonsingular sliding mode control. The simulation investigation is presented in Sect. 4. Section 5 concludes the paper.

\section{Model and dynamics of a Maglev system}

\subsection{System modeling}

Maglev train structure has two suspension systems: the upper car body between wheels and bogie and the lower between the bogie and car body. The suspension is necessary not only for isolating the car from vibrations and bumps but also to reduce the forces between wheels and rail. The bogie modeling is the key to the Maglev vehicle model [38]. In order to gain more insight into the behavior of the system, two majors factors are taken into account, the magnetic damping and stiffness of the air spring in the modeling which are the important parameters that determine the dynamic response and the stability of the Maglev system. These quantities in the equation of motion allow to gain more knowledge about the (nonlinear) dynamical behavior of the system for certain parameters and also enable the evaluation of their effects on the operation of the maglev $[39,40]$. For the sake of accuracy and for a judicious design of the Maglev train, various forms of active and passive damping mechanisms must be incorporated into the design to make the no steady-state operation stable [41]. The predominant approaches in the literature consider stiffness coefficient similar to the mechanical spring. The stability exists in the direction of positive stiffness if the reaction force acts to oppose perturbative displacements.

In order to analyze the dynamic of the maglev system, we consider the standard model structure presented in Fig. 1. This model is similar to those encountered in 
literature which consists of three subsystems: the spring system, guideway, and electromagnet system [29, 42]. The specificity of our approach compared to other existing works is that the influence of the spring system and the friction are not ignored. For more details, we refer the reader to [43-45]. We neglected the magnetic flux leakage and the edge effect which cannot significantly affect the dynamical behavior of the Maglev. We also consider only the vertical motion of the electromagnet.

Remark 1 Keep the levitation system steady is more difficult since the maglev force is inherently unstable, which leads to the dynamic characteristics performing diversity and complexity. Two approaches have been presented in literature such as the dynamics characteristics of the maglev vehicle structure and other way is focusing on controller design $[46,47]$. The big challenge is to study the impact of each parameter on the dynamic behavior of the device and to detect those whose influence is significant for the Maglev design. Unfortunately, by referring to the theoretical and experimental aspects, most strategies although satisfactory encountered in the literature remain qualitative (Hopf, bifurcation ...) which does not allow the rigorous establishment of the performance concepts of the Maglev system. An appropriate mathematical approach appears interesting and significantly helpful for us to understand the dynamical behavior of Maglev trains and to design relevant control laws.

Remark 2 The order of the Maglev will be high if all factors are taken into consideration which will be boring and the handing very difficult. For the sake of simplicity, we mention some important hypothesis considered in the analysis of this work: (1) Only vertical motion of the electromagnet is considered, namely, there is no displacement between the surface and the guideway. (2) the edge effect and the magnetic flux leakage are neglected, consequently, the flux is uniformly distributed in the air gap.

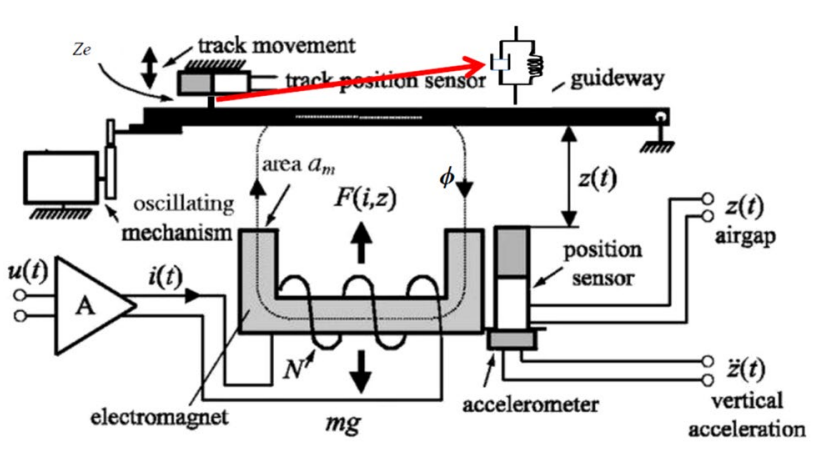

Fig.1 Configuration of the Maglev system
(3) the electromagnet develops an electromagnetic force which the origin is considered to be on the center of mass.

We derive the electromagnetic force as follows:

$F=\frac{d E_{m}}{d z}=\frac{d}{d z} \int e_{m} d v=\frac{d}{d z}\left(\frac{1}{2} B H V\right)=\frac{d}{d z}\left(\frac{B^{2} A z}{\mu_{0}}\right)=\frac{A B^{2}}{\mu_{0}}$

where $E_{m}$ represents the magnetic field energy of volume $V_{i} e_{m}$ is the magnetic field energy density; $\mathrm{A}$ is the magnetic pole area of the electromagnet; $\mu_{0}$ is air permeability and $\mathrm{F}$ denotes the electromagnetic attraction force. It is well known that the magnetic flux density can be expressed as $B=\frac{\mu_{0} N i(t)}{2 z(t)}$ and taking into account the Eq. (1), we finally derive the expression of the electromagnetic force generated by the electromagnet as follows:

$F(i, z(t))=\frac{\mu_{0} A N^{2}}{4}\left[\frac{i(t)}{z(t)}\right]^{2}$

where $z(t)$ is the magnet gap or the magnet displacement, $\mathrm{N}$ the number of turns and $i(t)$ the current of the solenoid coil. We suppose that components of the Maglev train are rigid bodies. Using the electrical and mechanical laws, it is found that the mechanical and the electrical dynamics are described by the following equations

$m \frac{d^{2} z(t)}{d t^{2}}+\beta(\dot{z}(t), z(t)) \frac{d z(t)}{d t}-m g=\frac{\mu_{0} A N^{2}}{4}\left[\frac{i(t)+\Delta i(t)}{z(t)+\Delta z(t)}\right]^{2}$

$\frac{\hbar}{2 z(t)} \frac{d i(t)}{d t}+R i(t)-\frac{\hbar}{z^{2}(t)} i(t) \frac{d z(t)}{d t}=u(t)$

$\Delta i(t)$ and $\Delta z(t)$ are the fluctuations of current and airgap when the Maglev moves on the guideway. At any point of the Maglev operation, the linearized electromagnetic force can be express as follows $[48,49]$

$\frac{\mu_{0} A N^{2}}{4}\left[\frac{i(t)+\Delta i(t)}{z(t)+\Delta z(t)}\right]^{2}=F(i, z(t))+k_{i} \Delta i(t)-K \Delta z(t)$

where $F(i, z(t))=\frac{\mu_{0} A N^{2}}{4}\left[\frac{i(t)}{z(t)}\right]^{2}, K=k_{i}=\frac{\mu_{0} A N^{2} i(t)^{2}}{2 z(t)^{2}}$ are the equivalent stiffnesses. Neglecting the fluctuation of the current in the electromagnetic force for the sake of simplicity, Eqs. (3) becomes

$$
\begin{aligned}
& m \frac{d^{2} z(t)}{d t^{2}}+\beta(\dot{z}(t), z(t)) \frac{d z(t)}{d t}+K \Delta z(t)-m g=\frac{\mu_{0} A N^{2}}{4}\left[\frac{i(t)}{z(t)}\right]^{2} \\
& \frac{\hbar}{2 z(t)} \frac{d i(t)}{d t}+R i(t)-\frac{\hbar}{z^{2}(t)} i(t) \frac{d z(t)}{d t}=u(t)
\end{aligned}
$$

Because very low damping is considered, the system is simplified to a constant damping coefficient: $\beta(\dot{z}(t), z(t))=\beta$. However, the equation is still nonlinear 
because of the nonlinear spring forces. $u(t)$ is the control voltage acting on both ends of the electromagnet. $m$ is the equivalent mass of the train body and the levitation chassis. $\mathrm{K}$ is the stiffness of the air spring. $z_{e}(t)$ the designed rating magnet gap $z(t), \beta$ the mechanical resistance and $a_{m}$ the area of magnetic the pole while $g$ is the gravity constant. $\Delta z(t)=z(t)-z_{e}$ is the magnet gab deviation. The simplified model of the slow medium maglev train can be obtained by introducing the new variables $y$ and $\omega$ as $z=y$ and $i=y \omega$ which lead to the following coupled systems:

$m \ddot{y}(t)+\beta \dot{y}(t)+K\left(y(t)-y_{e}\right)-m g=\frac{\hbar \omega^{2}(t)}{2}$

$\hbar \dot{\omega}(t)+R y(t) \omega(t)=u(t)$

where $\hbar=\frac{\mu_{0} N^{2} A}{2}$ denotes the force transmission coefficient.

Remark 3 It's easy to obtain the simplified model in [42, $50]$ by setting $\beta \approx 0$. In the following analysis, we recall that the mechanical resistance of the air spring is known and remains a fundamental characteristic of the Maglev described in [51-53].

\subsection{Equilibrium point and stability analysis}

The equilibrium points are solutions of the equations

$K\left(y(t)-y_{e}\right)-m g=\frac{\hbar \omega^{2}}{2}$

$R y(t) \omega(t)=u(t)$

\subsubsection{Case $u(t)=0$}

Denoting solution of Eqs. (8)-(9) by $\left(y_{0}, \omega_{0}\right)$, two distinct possibilities do exist.

If $y_{0}=0$, then, $\omega_{0}= \pm \sqrt{-2\left(\frac{m g}{\hbar}+\frac{K}{\hbar} y_{e}\right)} \cdot$ We then obtain two equilibrium points $\Omega_{1}\left(0, \sqrt{-2\left(\frac{m g}{\hbar}+\frac{k}{\hbar} y_{e}\right)}\right)$ and $\Omega_{2}\left(0,-\sqrt{-2\left(\frac{m g}{\hbar}+\frac{K}{\hbar} y_{e}\right)}\right)$ such that $m g \hbar+K \hbar y_{e} \leq 0$.

On the other hand, if $\omega_{0}=0$, the equilibrium point is therefore $\Omega_{3}\left(y_{e}+\frac{m g}{K}, 0\right)$.

The Jacobian matrix of the corresponding system is
$J=\left(\begin{array}{cc}K & -\hbar \\ R \omega_{0} & R y_{0}\end{array}\right)$

By considering the linearization around the $\Omega\left(y_{0}, \omega_{0}\right)$, the eigenvalues are solutions of the following characteristic equation, namely

$m \hbar \lambda^{3}+\left(m R y_{0}+\beta \hbar\right) \lambda^{2}+\left(K \hbar+\beta R y_{0}\right) \lambda+R\left(K y_{0}+\hbar \omega_{0}^{2}\right)=0$

We let $\beta_{1}=\frac{m R y_{0}+\beta \hbar}{m \hbar}, \beta_{2}=\frac{K \hbar+\beta R y_{0}}{m \hbar}$ and $\beta_{3}=\frac{R\left(K y_{0}+\hbar \omega_{0}^{2}\right)}{m \hbar}$.

For the equilibrium points $\Omega_{1}$ and $\Omega_{2}$, the following inequalities are verified $\beta_{1}{ }^{>} 0, \beta_{2}{ }^{>} 0$ and $\beta_{1} \beta_{2}{ }^{\prime} \beta_{3}$. According to the Routh-Hurwitz criteria, hence, these points are all unstable. And the other hand, considering $\Omega_{3}$, we have $\beta_{1}{ }^{2} 0, \beta_{2}{ }^{\prime} 0$ and $\beta_{1} \beta_{2}<\beta_{3}$. According to the Routh-Hurwitz criteria, the corresponding equilibrium point is stable.

Note: According to the stiffness criterion, it's easy to verify that the stability of the Maglev system can be expressed as follows:

a) Stable: $K>0$ b) Unstable: $K<0$

\subsubsection{Case $u(t)=0$}

Many authors have proven that numerous dynamical systems encountered in literature can depict a variable fixed point called AC equilibrium points [54] (the reader can also find an excellent text in Ref. [55]). This will allow a supplementary stringent aspect of many mechanicals systems which is submitted to small vibrations at a rest point.

Taking from (6) the parameter $\omega_{0}=\frac{u(t)}{R y_{0}}$, the equilibrium point $\left(y_{0}, \omega_{0}\right)$ is the solution of the function $f(y)$ defined as follows

$f\left(y_{0}\right)=K R^{2} y_{0}^{3}-R^{2}\left(K y_{e}+m g\right) y_{0}^{2}+k_{0} u^{2}(t)=0$

where $k_{0}=-\frac{\hbar}{2}$. The first derivative of $f\left(y_{0}\right)$ is given by

$\frac{d f\left(y_{0}\right)}{d y_{0}}=3 K R^{2} y_{0}^{2}-2 R^{2}\left(K y_{e}+m g\right) y_{0}$

The solutions of (12) are defined as follows

$\frac{d f(y)}{d y}=0 \Rightarrow y_{10}=0, \quad y_{20}=\frac{2\left(K y_{e}+m g\right)}{3 K}$,

Let's define a continuous smooth function $F$ such that

$F=f\left(y_{01}\right) \times f\left(y_{02}\right)=\frac{\hbar^{2}}{4} u^{4}-\frac{1}{27} \frac{\hbar^{2} u^{2} R^{2}\left(K y_{e}+m g\right)^{3}}{K^{2}}$

a. If $F^{>} 0$ that is $\frac{1}{4} u^{2>} \frac{1}{27} \frac{R^{2}\left(K y_{e}+m g\right)^{3}}{K^{2}}$ 
$f\left(y_{0}\right)$ has one solution, consequently one equilibrium point. Finally, the solution obtained is

$$
\begin{aligned}
& y_{0}=\frac{1}{3} \frac{K y_{e}+m g}{K}+\left(-\frac{1}{27}\left(y_{e}+\frac{m g}{K}\right)^{3}-\frac{\hbar u^{2}}{2 K R^{2}}+\frac{1}{2} \sqrt{\delta}\right)^{1 / 3} \\
& +\left(-\frac{1}{27}\left(y_{e}+\frac{m g}{K}\right)^{3}-\frac{\hbar u^{2}}{2 K R^{2}}-\frac{1}{2} \sqrt{\delta}\right)^{1 / 3}
\end{aligned}
$$

b. If $F=0$ that is $u^{2}=\frac{4}{27} \frac{R^{2}\left(K y_{e}+m g\right)^{3}}{K^{2}}$

We have two equilibrium points as follows

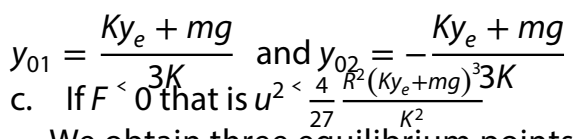

We obtain three equilibrium points

$$
\begin{aligned}
& y_{001}=\frac{K y_{e}+m g}{3 K}(1+\cos (\theta)) \\
& y_{002}=\frac{K y_{e}+m g}{3 K}\left(1+\cos \left(\theta+\frac{2 \pi}{3}\right)\right) \\
& y_{003}=\frac{K y_{e}+m g}{3 K}\left(1+\cos \left(\theta+\frac{4 \pi}{3}\right)\right)
\end{aligned}
$$

With

$$
\theta=\frac{1}{3} \arctan \left(\frac{\sqrt{-\delta}}{-\frac{2}{27}\left(y_{e}+\frac{m g}{K}\right)^{3}-\frac{\hbar u}{2 K R^{2}}}\right)
$$

These results are relaxed and proven at the end (see the appendix).

\subsection{Amplitude response}

In the aims to observe the evolution of the amplitude response of the Maglev system, an analytical expression of displacement $z(t)$ and the current $i(t)$ flowing through the electromagnet are for high interest. In order to solve the set of Eqs. 6 and 7, let us make the change of variables:

$y(t)=b+\varphi(t)$

where $b$, and $\varphi(t)$ are constant and smooth function respectively to be determined. Inserting Eqs. (20) into Eqs. (6)-(7), one obtains

$m \ddot{\varphi}(t)+\beta \dot{\varphi}(t)+K\left(b-y_{e}(t)\right)-m g=\frac{\hbar}{2} \omega^{2}(t)$ $\hbar \dot{\omega}(t)+R b \omega(t)+R \varphi(t) \omega(t)=u(t)$

Setting $\omega(t)$ weak, then the product $\varphi(t) \omega(t)$ is too weak. Letting $u(t)=U_{m} \sin (\Omega t)$, it's obvious to obtain from (22) that

$\omega(t)=C \exp \left(-\frac{R b}{\hbar} t\right)+\frac{U_{m}}{R^{2} b^{2}+\Omega^{2} \hbar^{2}}(R b \sin (\Omega t)-\Omega \hbar \cos (\Omega t)$

If we seek for harmonic solution, we set $C=0$ and derive the following solutions

$S_{1}=\frac{R b}{R^{2} b^{2}+\Omega^{2} \hbar^{2}}$ and $S_{2}=\frac{\Omega \hbar}{R^{2} b^{2}+\Omega^{2} \hbar^{2}}$

and substituting (24) into (20), one has

$b=y_{e}+\frac{m g}{K}+\frac{\hbar\left(S_{1}^{2}+S_{2}^{2}\right)}{2 K\left(R^{2} b^{2}+\Omega^{2} \hbar^{2}\right)}=y_{e}+\frac{m g}{K}+\frac{\hbar U_{m}^{2}}{2 K\left(R^{2} b^{2}+\Omega^{2} \hbar^{2}\right)}$

Equation (25) leads to the constraints

$$
\begin{gathered}
2 K R^{2} b^{3}-2 R^{2}\left(K y_{e}+m g\right) b^{2}+2 K \Omega^{2} \hbar^{2} b \\
-2 K \Omega^{2} \hbar\left(K y_{e}+m g\right)-0.5 \hbar U_{m}^{2}=0
\end{gathered}
$$

We set $y_{0}^{\prime}=\frac{1}{3}\left(y_{e}+\frac{m g}{K}\right), P=\left(y_{0}^{\prime 2}-\hbar^{2} \frac{\Omega^{2}}{3 R^{2}}\right)^{3}, S=y_{0}^{\prime 2}+\frac{y_{0}^{\prime} h^{2} \Omega^{2}}{R^{2}}+\frac{1}{8} \frac{\hbar U_{m}^{2}}{K R^{2}}$ and.

$Q(S, P)=S^{2}-P^{2}$. The quantity $Q(S, P)$ is called the discriminant. The sign of this quantity clearly defines the complete behavior of the Maglev train at the particular moment.

From Cardan method, the Eq. (26) admits the solution according to:

a. If $Q(S, P)^{\prime} 0$, we have only one real solution

$$
b=y_{0}+\left(s-\sqrt{S^{2}-P^{2}}\right)^{1 / 3}+\left(s+\sqrt{S^{2}-P^{2}}\right)^{1 / 3}
$$

b. If $Q(S, P)=0$, we have two solutions $b=y_{0}+S^{1 / 3}$ or $b=y_{0}-S^{1 / 3}$

c. If $Q(S, P),<0$ we have three solutions

$$
\begin{aligned}
& b=y_{0}+2 P^{1 / 3} \cos (\vartheta) ; b=y_{0}+2 P^{1 / 3} \cos \left(\vartheta+\frac{2 \pi}{3}\right) ; \\
& b=y_{0}+2 P^{1 / 3} \cos \left(\vartheta+\frac{4 \pi}{3}\right)
\end{aligned}
$$

with $\vartheta=\frac{1}{3} \arctan \left(\frac{\sqrt{P-S^{2}}}{S}\right)$. 
Finally, the analytical solution of the electromechanical system (6)-(7) is as follows: where $\left(a_{1}, a_{2}\right)$ and $\left(a_{01}, a_{02}\right)$ denotes the constants coefficients of the trigonometric function

$$
\begin{aligned}
& y(t)=y_{e}+\frac{m g}{K}+\frac{\hbar U_{m}^{2}}{2 K\left(R^{2} b^{2}+\Omega^{2} \hbar^{2}\right)}+\Sigma_{0}\left(\left(R^{2} b^{2}+\Omega^{2} \hbar^{2}\right)\left(K-4 m \Omega^{2}\right)-4 R b \hbar \beta \Omega^{2}\right) \cos (2 \Omega t) \\
& +2 \Omega \Sigma_{0}\left(R b \hbar\left(K-4 m \Omega^{2}\right)+\beta\left(R^{2} b^{2}+\Omega^{2} \hbar^{2}\right)\right) \sin (2 \Omega t)
\end{aligned}
$$

$\omega(t)=\frac{U_{m}}{R^{2} b^{2}+\Omega^{2} \hbar^{2}}(R b \sin (\Omega t)-\Omega \hbar \cos (\Omega t))$

where

$$
\Sigma_{0}=-\frac{\hbar U_{m}^{2}}{4\left[\left(K-4 m \Omega^{2}\right)^{2}+4 \beta^{2} \Omega^{2}\right]\left(R^{2} b^{2}+\Omega^{2} \hbar^{2}\right)^{2}}
$$

Comparing (20) and (29), we identify the constant parameter defined as

$b=y_{e}+\frac{m g}{K}-\frac{\hbar U_{m}^{2}}{2 K\left(R^{2} b^{2}+\Omega^{2} \hbar^{2}\right)}$

Taking into consideration Eq. (29), the solution can be defined as

$$
\left\{\begin{array}{l}
y(t)=z_{0}+z_{m} \cos \left(2 \Omega t-\varphi_{z}\right) \\
i(t)=l_{1} \cos \left(\Omega t-\varphi_{1}\right)+l_{3} \cos \left(3 \Omega t-\varphi_{2}\right)
\end{array}\right.
$$

$z_{0}$ is the initial position of the maglev which stands as a mean value of $z(t) \cdot z_{m}$ is the amplitude of $z(t) . I_{1}$ and $I_{2}$ are the amplitude of the current for the fundamental and for the third harmonic. $\varphi_{z^{\prime}} \varphi_{1}$ and $\varphi_{2}$ denote the corresponding phase for the position $z(t)$ and the current $i(t)$ respectively. It's straightforward to obtain after some developments the following results:

$$
\begin{aligned}
& z_{0}=y_{e}+\frac{m g}{K}+\frac{\hbar U_{m}^{2}}{2 K\left(R^{2} b^{2}+\Omega^{2} \hbar^{2}\right)} \\
& z_{m}=\Sigma_{0} \sqrt{\left(\left(R^{2} b^{2}+\Omega^{2} \hbar^{2}\right)\left(K-4 m \Omega^{2}\right)-4 R b \hbar \beta \Omega^{2}\right)^{2}+4 \Omega^{2}} \\
& \varphi_{z}=\arctan \left(\frac{\left(\left(R^{2} b^{2}+\Omega^{2} \hbar^{2}\right)\left(K-4 m \Omega^{2}\right)-4 R b \hbar \beta \Omega^{2}\right)}{2 \Omega\left(R b \hbar\left(K-4 m \Omega^{2}\right)+\beta\left(R^{2} b^{2}+\Omega^{2} \hbar^{2}\right)\right)}\right)
\end{aligned}
$$

$$
I_{1}=\sqrt{\left(\frac{a_{2} a_{01}}{2}-\frac{a_{1} a_{02}}{2}-a_{02} y_{0}\right)^{2}+\left(\frac{a_{2} a_{02}}{2}-\frac{a_{1} a_{01}}{2}-a_{01} y_{0}\right)^{2}}
$$

$$
I_{3}=\sqrt{\left(\frac{a_{1} a_{01}}{2}+\frac{a_{2} a_{02}}{2}\right)^{2}+\left(\frac{a_{1} a_{02}}{2}+\frac{a_{2} a_{01}}{2}\right)^{2}}
$$

$(\cos (2 \Omega t), \sin (2 \Omega t))$ and $(\sin (2 \Omega t), \cos (2 \Omega t))$ referring to the solutions $y(t)$ and $\omega(t)$ respectively.

From Eq. (33), we can highlight two essential observations: (a) the presence of harmonic currents is one of factors which have the most influence on the technical design of the electromagnet or the associated generator, more particularly with reference to the waveform. These currents cause distortions that move the wave profile away from the ideal sine reference. Accordingly, in this article, the system's parameters should properly choose to eliminate the third harmonics or to render this higher order component negligible and thus attenuate their effect. (b) The mechanical frequency vibration is probably twice that of the source voltage.

\section{Simulations validation of the theory}

Let consider the physical parameters of Maglev system as presented in Refs. $[21,36,38]$ which are listed in Table 1.

For the sake of clarity, we choose the control input voltage as $u(t)=220 \sqrt{2} \sin (2 \pi f t)$ and the radian frequency is $\omega=2 \pi f$ where $f$ is chosen as $50 \mathrm{~Hz}$. Now, let us analyze the dynamical behavior of the displacement and the current flowing in the coil. The numerical method used to find the desired solutions is fixed step size fourthorder Runge-Kutta algorithm with step $67 \times 10^{-5}$. The initial conditions considered are $z(0)=z_{0} ; \dot{z}(0)=0$ and $i(0)=z(0) \omega(0)=0$. We derive the corresponding
Table 1 Parameters description

\begin{tabular}{lll}
\hline Parameters & Description & Value \\
\hline$m$ & Mass of the maglev module & $800 \mathrm{~kg}$ \\
$A$ & Area of the magnetic pole & $0.0183 \mathrm{~m}^{2}$ \\
$\mu_{0}$ & Space permeability & $4 \pi .10^{-7} \mathrm{Hm}^{-1}$ \\
$R$ & Resistance of the coil & $0.56 \Omega$ \\
$L$ & Length of electromagnet & 1.36 \\
$N$ & Number of turns of the electromagnet & 360 \\
$K$ & Stiffness of air spring & $52.10^{4} \mathrm{~N} / \mathrm{mm}$ \\
$\beta$ & Mechanical resistance & $2.3 \mathrm{Ns} / \mathrm{m}$ \\
$g$ & Gravitaty acceleration & $9.81 \mathrm{~N} / \mathrm{kg}$ \\
\hline
\end{tabular}


Fig. 2 Time evolution of the system, a Displacement $y(t)$ and $\mathbf{b}$ Current $i(t)$

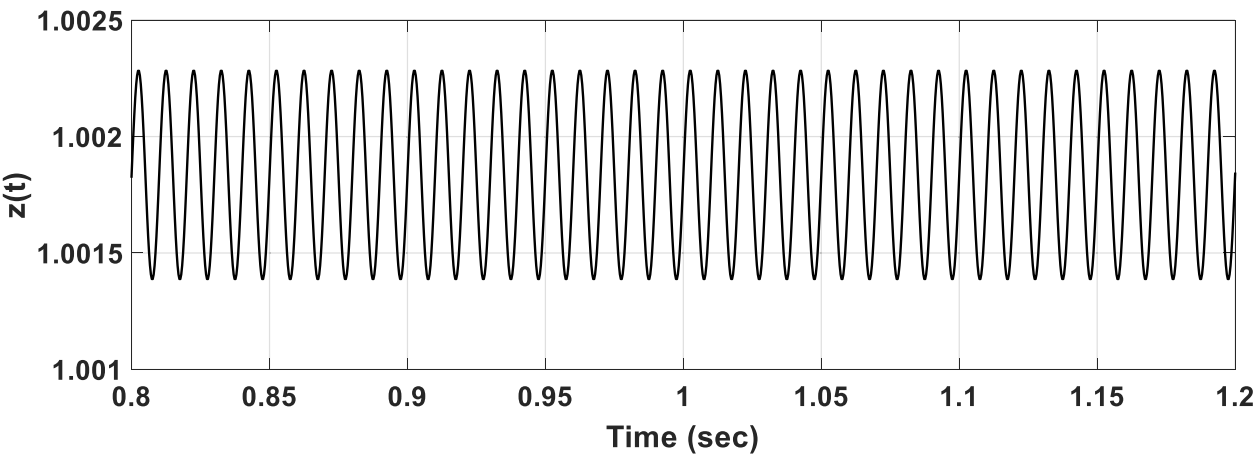

(a)

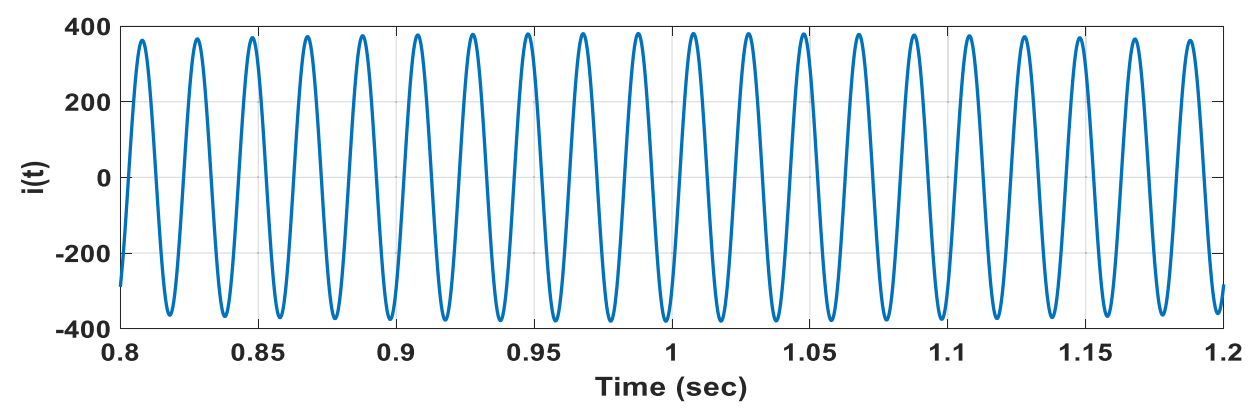

(b)
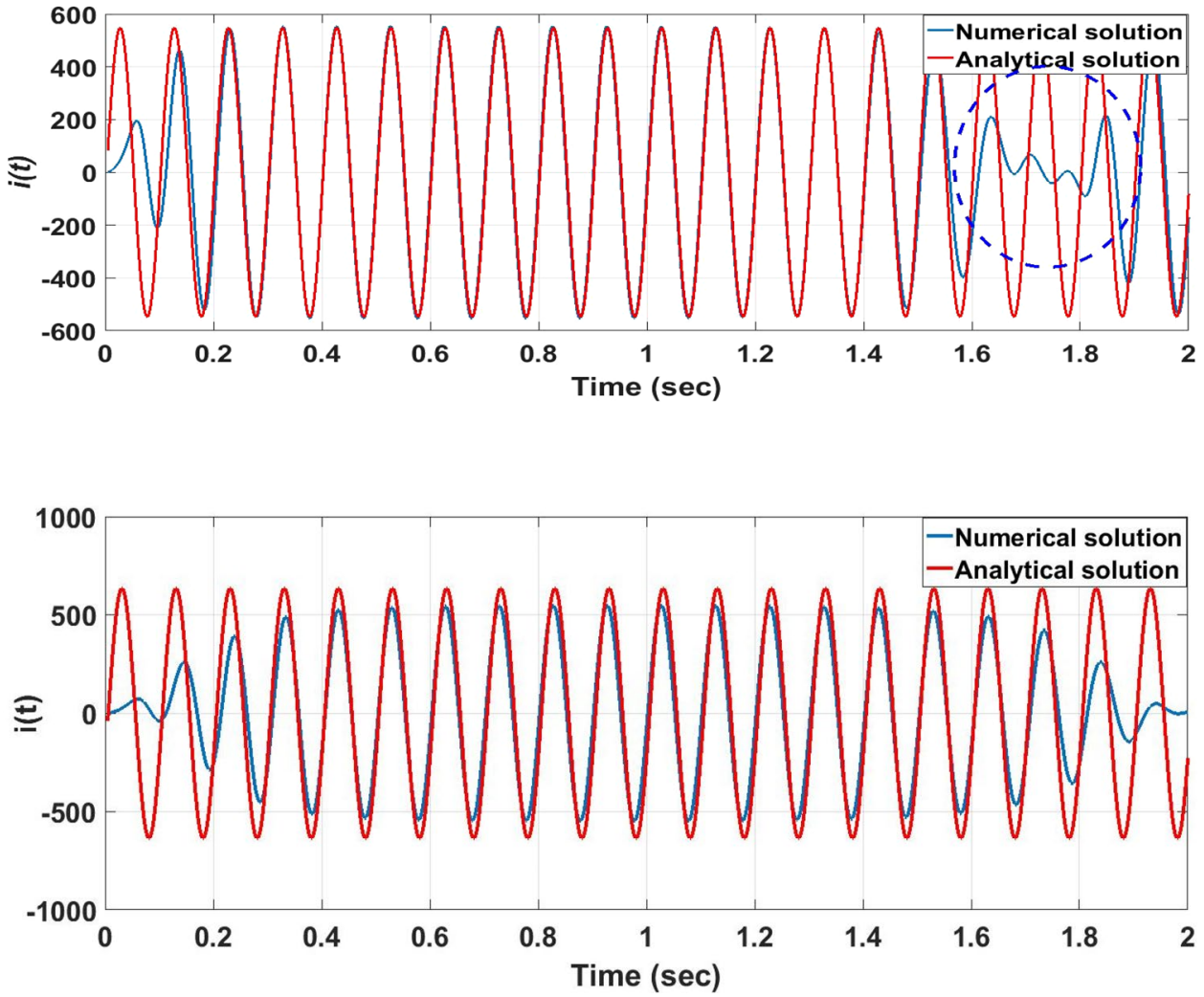
numerical solutions of the differential Eqs. (3.1) and (3.2) showed on Fig. 2a, b. We clearly observe that the oscillation frequency is twice the one of the current.

In order to investigate the effects of each parameter on the behavior of the system, let us depict Fig. 3. The red curve represents the numerical solution obtained from Eq. (19) and the blue one is the analytical solution. We notice that for weak values of the vibration frequency, the two curves are similar. A disparity appears in the gap $[1.5 ; 1.85]$ and reveals an irregular behavior of the electromagnet in the dynamics of the overall system. The mathematical approximation of the Eq. (19) does not take into consideration this discontinuity. This behavior is observed for $m=520 \mathrm{Kg}$. Figure 3 shows the same curves for $m=620 \mathrm{Kg}$. We obviously point out that the solution obtained remains rigorously true in a sufficient time interval to be able to make a complete analysis of the Maglev. From Fig. 2a, it appears that the great variation of the displacement may destroy the performance of the maglev system. The current that is given to the electric coils of the guideway will be alternating in nature. Thus the polarity of the coils will be changing in period. Figures 3 and 4 depict
Fig. 5 Displacement responses of the guideway a Numerical solution, b Analytical solution

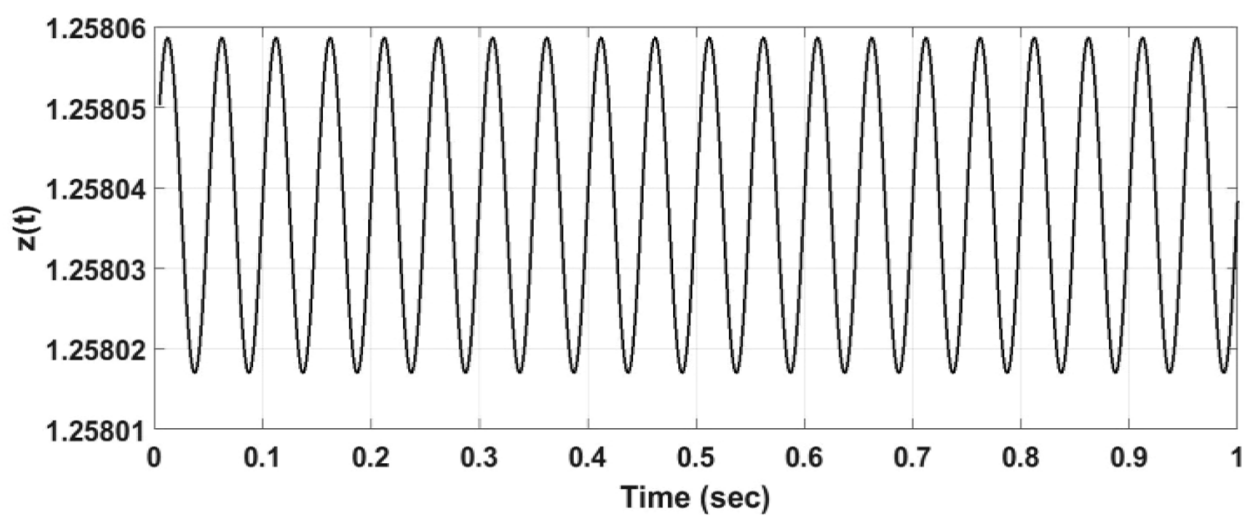

(a)

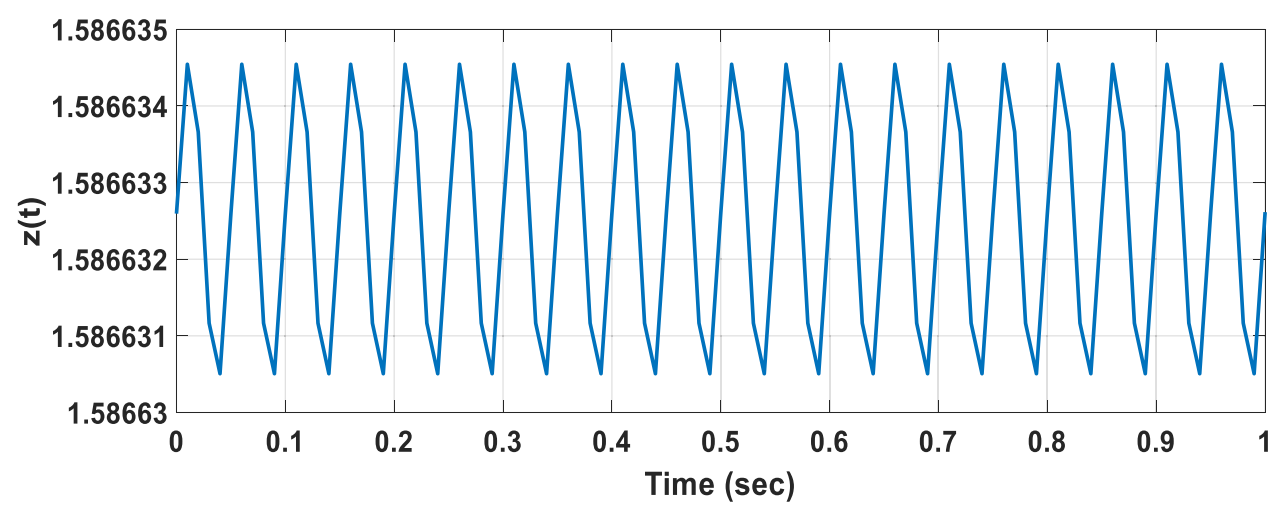

(b)

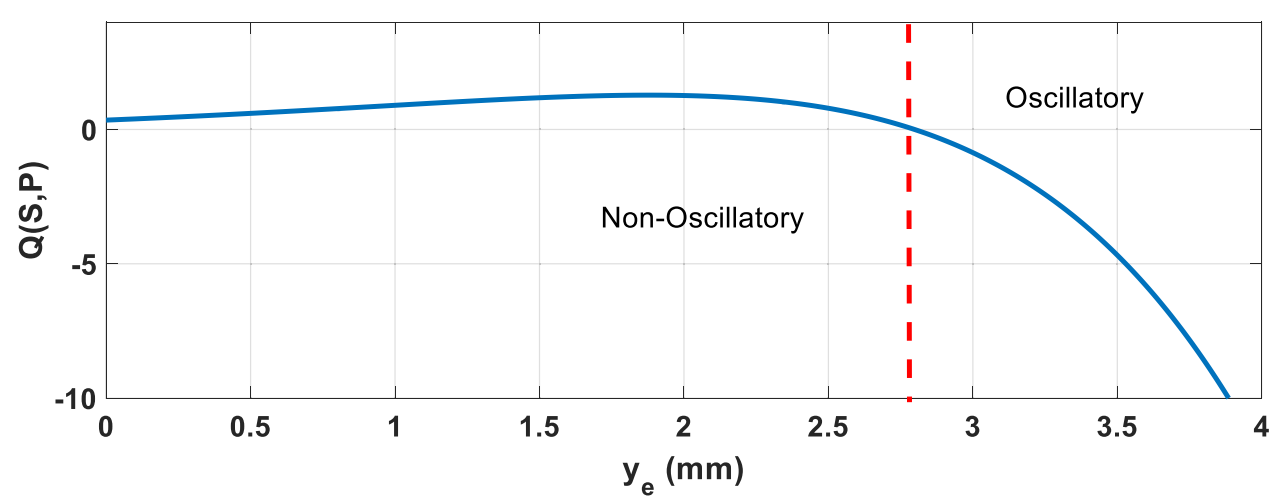

Fig. 6 Oscillatory and nonoscillatory zone according the initial selection of the displacement $\mathrm{y}_{\mathrm{e}}(\mathrm{mm})$ 
respectively, the numerical solution of the displacement and the analytical solution from Eq. (19). We observe a perfect agreement between these curves which confirms the performance of the general proposed solution within a suitable interval. In addition, one of the important aspects of this study is also to know the parameter which would considerably reduce the harmonics in the signals of the Maglev, in fact, without loss of generality, from Eqs. (27) and (31), it clearly appears that the relevant design of the value of air spring $(\mathrm{K})$ of the electromagnet assigns considerably the dynamical properties of the vehicle and then becomes fundamental to eliminate the harmonic in the Maglev system. Hence, the change causes a pull force for the train in the front and when it is added to this force, the magnetic field behind the train adds more forward thrust. Figures $5 a$, b, present the numerical and analytic solution of the displacement. These results are obtained for the small values of the vibrating frequencies $(10-30 \mathrm{~Hz})$, when the frequency increases, the maglev system become boring and the control problem takes place. One important issue to improve technologically the Maglev system is the selection of a device which enables slow variation of the displacement of the air gap, Fig. 6 shows the zone in which the oscillations appears.

\section{Preliminaries}

\subsection{Control scheme description and objective}

To deal with the solution of the Maglev control, the electromechanical Eqs. (6)-(7) can be put in the canonical form of differential equation. By letting $y(t)=x_{1}(t), \dot{x}_{1}(t)=x_{2}(t), x_{3}(t)=\omega, g_{0}=\frac{K}{m} x_{1 e}+g$ and $x_{1 e}=y_{e}$, we obtain

$\dot{x}_{1}=x_{2}$

$\dot{x}_{2}=\frac{K}{m} x_{1}-\frac{\beta}{m} x_{2}-\frac{\hbar}{2 m} x_{3}^{2}+g_{0}$

$\dot{x}_{3}=-\left(\frac{R}{L}\right) x_{1} x_{3}+\left(\frac{u(t)}{L}\right)$

Let consider $x_{1 s}, x_{2 s}$ and $x_{3 s}$ be the target values of $x_{1}, x_{2}$ and $x_{3}$ respectively. It obvious to note that from Eqs. (39), the equilibrium point is $x_{0}=\left(x_{10}, 0, x_{30}\right)^{T}$, where $x_{30}$ satisfies $x_{30}=\sqrt{-2\left(\frac{k}{\hbar} x_{10}+\frac{g_{0}}{\hbar}\right)}$. The control objective is to design a robust control $u(t)$ which will drive the states $x_{1}, x_{2}$ and $x_{3}$ to its desired constant values $x_{1 s}, x_{2 s}$ and $x_{3 s}$ respectively which can be expressed in the mathematical form by

$\lim _{t \rightarrow \infty}\left\|\left(x(t)-x_{0}\right)^{T}\right\|=0$

\subsection{Problem formulation}

We first introduce the change coordinators as follows:

$\varepsilon_{1}=x_{1}-x_{10}$

$\varepsilon_{2}=x_{2}$

$\varepsilon_{3}=\frac{K}{m} x_{1}-\frac{\beta}{m} x_{2}+\frac{\hbar}{2 m} x_{3}^{2}+g_{0}$

It's obvious to remark that when $\varepsilon_{1}, \varepsilon_{2}, \varepsilon_{3}$ are driven to zero as $t \rightarrow \infty$, then $x_{1}$ will converge to $x_{10}, x_{2}$ will converge to zero, and $x_{3}$ will converge to $x_{30}=\sqrt{-2\left(\frac{K}{\hbar} x_{10}+\frac{g_{0}}{\hbar}\right)}$.

The first derivative of Eq. (41) leads to the following
Fig. 7 Control block diagram of the Maglev controlled system

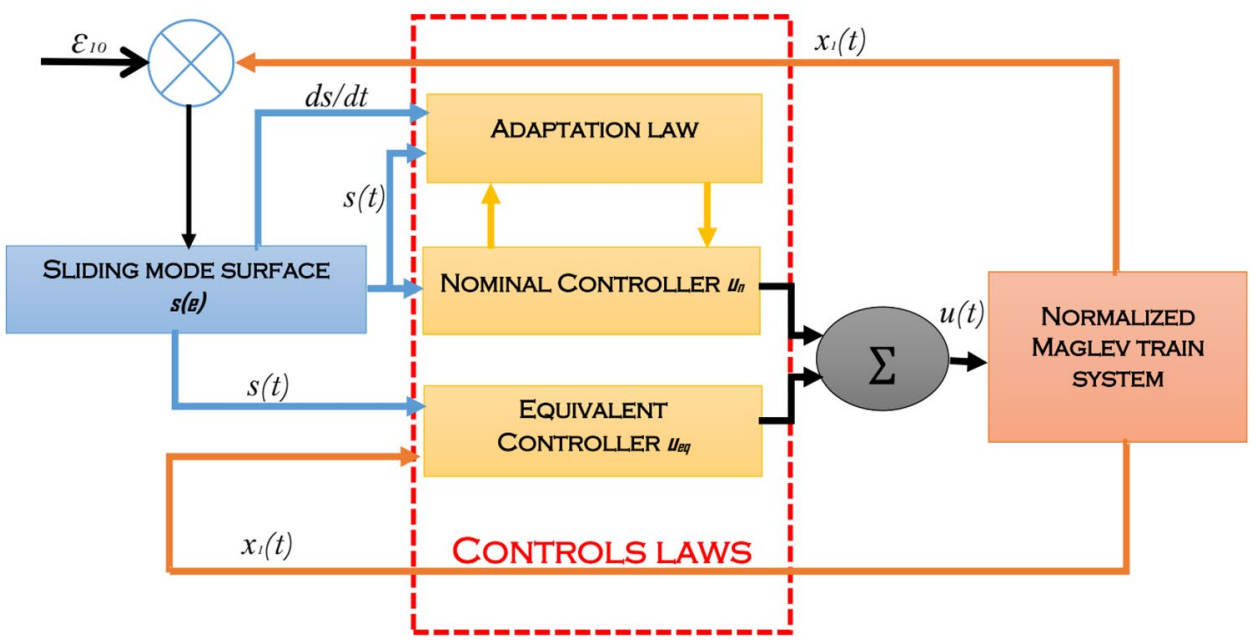


$\dot{\varepsilon}_{1}=\dot{x}_{1}$

$\dot{\varepsilon}_{2}=\dot{x}_{2}$

$\dot{\varepsilon}_{3}=h(\varepsilon)+g(\varepsilon) u(t)$

where $h(\varepsilon)$ and $g(\varepsilon)$ are functions expressed as follows:
We design the control law as

$u=g^{-1}(\varepsilon, t)\left(u_{e q}+u_{n}\right)=\tilde{u}_{e q}+\tilde{u}_{n}$

$u_{e q}$ can be designed as

$h(\varepsilon)=\left(\frac{K}{m}+\left(\frac{\beta}{m}\right)^{2}\right) \varepsilon_{2}-\frac{\beta K}{m^{2}}\left(\varepsilon_{1}+x_{10}\right)-\frac{g_{0} K}{m}-\frac{m}{k}\left(\frac{\beta \hbar}{2 m^{2}}+\frac{R \hbar}{m L}\left(\varepsilon_{1}+x_{10}\right)\right)\left(\frac{K}{m}\left(\varepsilon_{1}+x_{10}\right)-\frac{\beta}{m} \varepsilon_{2}-\varepsilon_{3}+g_{0}\right)$

$g(\varepsilon)=\frac{\hbar}{m L} \sqrt{\left(\frac{K}{m}\left(\varepsilon_{1}+x_{10}\right)-\frac{\beta}{m} \varepsilon_{2}-\varepsilon_{3}+g_{0}\right)}$

\subsection{Robust sliding mode control design}

Let consider Eqs. (42) of uncertain nonlinear maglev system as follows

$\dot{\varepsilon}_{1}=\varepsilon_{2}$

$\dot{\varepsilon}_{2}=\varepsilon_{3}$

$\dot{\varepsilon}_{3}=h(\varepsilon)+g(\varepsilon) u(t)+\Lambda(t)$

where $\Lambda(t)$ is the bounded disturbance that affects the dynamic of the maglev system. We assume that $\Lambda(t) \in L_{2}$, that is $\int_{0}^{\infty} \Lambda^{T}(t) \Lambda(t) d t \leq 0$ and the derivative $\Lambda(t)$ is bounded, that is $\dot{\Lambda}(t)^{<} \Delta$.

Remark 2. The diagram overview of the control is illustrated in Fig. 7. The full order adaptive nonsingular sliding mode control is considered in the scheme under the exogenous disturbances.

A sliding surface is chosen as

$s(\varepsilon, t)=\dot{\varepsilon}_{3}+\theta_{3} \varepsilon_{3}+\theta_{2} \varepsilon_{2}+\theta_{1} \varepsilon_{1}$

$\theta_{1}, \theta_{2}$ and $\theta_{3}$ are constants which are selected such that the polynomial $p^{3}+\theta_{3} p^{2}+\theta_{2} p+\theta_{1}$ is Hurwitz. The aim of this part is to develop an adaptive nonsingular sliding mode controller such that the closed loop (44) states converge to zero in limited time. $u_{e q}=-h(\varepsilon)-\theta_{3}\left(\varepsilon_{3}\right)^{\frac{1}{\lambda}}-\theta_{2}\left(\varepsilon_{2}\right)^{\frac{1}{\lambda}}-\theta_{1}\left(\varepsilon_{1}\right)^{\frac{1}{\lambda}}$

$u_{n}$ can be taken as

$\dot{u}_{n}+t u_{n}=v$

where $v$ is designed as

$v=-\alpha \frac{s}{|s|+\varepsilon^{\prime}}-\kappa \operatorname{sgn}(s(t))$

where $u_{n}(0)=0, \varepsilon^{\prime}$ is a small positive number and $\kappa$ the positive constant which is automatically update by the designer according to the following law:

$\frac{d}{d t} \kappa=\gamma_{0}|s(\varepsilon, t)|$

where $\gamma_{0}>0$ is adaptation gain

Theorem The system (44) reaches and converges to the sliding surface in limited time if the selected sliding surface (45) and the control law as above are considered.

Proof From (45), we design the sliding surface as:

$$
\begin{aligned}
s(\varepsilon, t) & =\dot{\varepsilon}_{3}+\theta_{3}\left(\varepsilon_{3}\right)^{\frac{1}{\lambda}}+\theta_{2}\left(\varepsilon_{2}\right)^{\frac{1}{\lambda}}+\theta_{1}\left(\varepsilon_{1}\right)^{\frac{1}{\lambda}} \\
& =f(\varepsilon, t)+\alpha(\varepsilon, t) u(t)+\Lambda(t)+\theta_{3}\left(\varepsilon_{3}\right)^{\frac{1}{\lambda}}+\theta_{2}\left(\varepsilon_{2}\right)^{\frac{1}{\lambda}}+\theta_{1}\left(\varepsilon_{1}\right)^{\frac{1}{\lambda}}
\end{aligned}
$$

Take into consideration, (46), (47) and (50), we obtain

$$
\begin{aligned}
& s(\varepsilon, t)=\dot{\varepsilon}_{3}+\theta_{3} \varepsilon_{3}+\theta_{2} \varepsilon_{2}+\theta_{1} \varepsilon_{1} \\
& =f(\varepsilon, t)+\alpha(\varepsilon, t) u(t)+\Lambda(t)+\theta_{3} \varepsilon_{3}+\theta_{2} \varepsilon_{2}+\theta_{1} \varepsilon_{1} \\
& =f(\varepsilon, t)+\alpha(\varepsilon, t)\left[-\alpha^{-1}(\varepsilon, t) f(\varepsilon, t)-\theta_{3} \varepsilon_{3}-\theta_{2} \varepsilon_{2}-\theta_{1} \varepsilon_{1}-\alpha^{-1}(\varepsilon, t) u_{n}\right]+\Lambda(t)+\theta_{3} \varepsilon_{3}+\theta_{2} \varepsilon_{2}+\theta_{1} \varepsilon_{1} \\
& =u_{n}+\Lambda(t)
\end{aligned}
$$


The derivative of (52) gives

$$
\begin{aligned}
& \dot{s}(\varepsilon, t)=\dot{u}_{n}+\frac{d}{d t} \Lambda(t) \\
& =v-t u_{n}+\frac{d}{d t} \Lambda(t) \\
& =-\alpha \frac{s}{|s|+\varepsilon}-\kappa \operatorname{sign}(s)-t u_{n}+\frac{d}{d t} \Lambda(t)
\end{aligned}
$$

The Lyapunov function candidate can be defined as

$V(t)=\frac{1}{2} s^{2}+\frac{1}{2}(\kappa(t)-\kappa(0))^{2}$

Substituting (53) into (54), one obtains of the proposed control scheme. The external disturbance representing time-varying parameter uncertainties is considered as $\Lambda(t)=5.10^{-3} \sin (0.5 t)$. The initial conditions of the differential maglev Eqs. (44) are $\left(10^{-3}, 0.8,0.4,0.6,10^{-3}\right)$. The results of the proposed scheme are validated using Routh Hurwitz criteria. We first select the parameters of the polynomial as follows $\alpha=35, \varepsilon=10^{-3}, \theta_{1}=100, \theta_{2}=121, \theta_{3}=151, \gamma_{0}=-0.01$ It's obvious to mention for this case that the Hurwitz criteria is not satisfied. With this condition the maglev train remains uncontrollable. Figures $8 \mathrm{a}, \mathrm{b}, \mathrm{c}$ present the surface and the states $\varepsilon_{1}(t)$ and $\varepsilon_{2}(t)$ when the control is not effective.

For the sake of simplicity, we choose $\lambda_{i}=1,(i=1,2,3)$ which confers to the stability of the maglev system

$$
\begin{aligned}
& \dot{V}(t)=s(\varepsilon, t)\left(-\alpha \frac{s(\varepsilon, t)}{|s(\varepsilon, t)|+\varepsilon}-\kappa \operatorname{sign}(s(\varepsilon, t))-t u_{n}+\frac{d}{d t} \Lambda(t)\right)+\dot{\kappa}(\kappa(t)-\kappa(0)) \\
& =-\alpha \beta|s(\varepsilon, t)|-\kappa|s(\varepsilon, t)|-t u_{n} s(\varepsilon, t)+s(\varepsilon, t) \frac{d}{d t} \Lambda(t)+\gamma_{0}|s(\varepsilon, t)| \kappa(t)-\gamma_{0}|s(\varepsilon, t)| \kappa(0) \\
& =-\alpha \beta|s(\varepsilon, t)|-\left(1-\gamma_{0}\right) \kappa(t)|s(\varepsilon, t)|-t u_{n} s(\varepsilon, t)+s(\varepsilon, t) \frac{d}{d t} \Lambda(t)-\gamma_{0}|s(\varepsilon, t)| \kappa(0) \\
& =-\alpha \beta|s(\varepsilon, t)|-\left(1-\gamma_{0}\right) \kappa(t)|s(\varepsilon, t)|-t u_{n} s(\varepsilon, t)-\left(\gamma_{0} \kappa(0)-\operatorname{sign}(s(\varepsilon, t)) \frac{d}{d t} \Lambda(t)\right)|s(\varepsilon, t)| \\
& \prec-\alpha \beta|s(\varepsilon, t)|-\left(1-\gamma_{0}\right) \kappa(t)|s(\varepsilon, t)|-t u_{n} s(\varepsilon, t)-\left(\gamma_{0} \kappa(0)-\operatorname{sign}(s(\varepsilon, t)) \Delta\right)|s(\varepsilon, t)|
\end{aligned}
$$

where $\frac{s}{|s|+\varepsilon}=\beta^{\prime} \operatorname{sign}(s)$ with $0^{<} \beta^{\prime<} 1$.

We make the following considerations,

$\left(1-\gamma_{0}\right) \kappa(t)>0$ provide that. $\gamma_{0}<1$

$\gamma_{0} \kappa(0)-\operatorname{sign}(s(\varepsilon, t)) \Delta>0$ then $|\Delta|<\gamma_{0} \kappa(0)$

Expression (55) leads to $\dot{V}(t)^{<} 0$ which completes the proof of the theorem.

Remark 3 In order to obtain the expression of $u_{n}$, let consider the Laplace transform of Eqs. (48)

$(s+t) u_{n}(s)=v(s) \rightarrow u_{n}(s)=\frac{1}{s+t} v(s)$

We also assume that $u_{n}(0)=0$ and for $t=0$, we have

$\frac{u_{n}(s)}{v(s)}=\frac{1}{s}$

\section{Numerical simulation investigating robust control of Maglev train}

The simulations are based on Fig. 7. The values on the Tab. 1 are considered in the numerical investigations. Matlab simulations are performed to verify the performance
(Hurwitz criteria is verified). For convenience, from Fig. 6, we select arbitrarily $x_{10}$ close to $y_{e}$ in order to remain in the oscillatory region such that

$\left|y_{e}-x_{10}\right| \leq 10 \% m m$.

Let consider the initial position as $y_{e}=10 \mathrm{~mm}$, the target air gap is taken as $\bar{y}=3.7 \mathrm{~mm}$. The simulation results can be observed in Fig. 9 a, b, c. From Fig. 9a, we can observe that the displacement of the rail and the air gap reaches stability. From Fig. 9 b, c, one can observe that, after a transient period, the current and the speed become stable and converge to zero which is in good agreement with our theoretical results. It is important to recall that the convergence to zero of the state variables describing the current through the coil doesn't imply that the quantity vanishes, but it also shows the great reduction of the harmonic in the maglev system. Figures 10 and 11 depict the sliding surface $s(t)$ and the control gain $k(t)$ respectively. It is observed that the control effort which is measured by $\int_{0}^{\infty} u(t) d t$ is weak and therefore can be easily implemented in real time. From the above results, the response system of the Maglev system has a good control performance. We can clearly observe that the proposed sliding mode control helps to stabilize the maglev on the rail at around $9.5 \mathrm{~s}$ despite perturbations. Let's point out some promising results obtained in the literature using the relevant control 
Fig. 8 Time of the sliding surface and the state variables $\varepsilon_{1}(t)$ and $\varepsilon_{2}(t)$

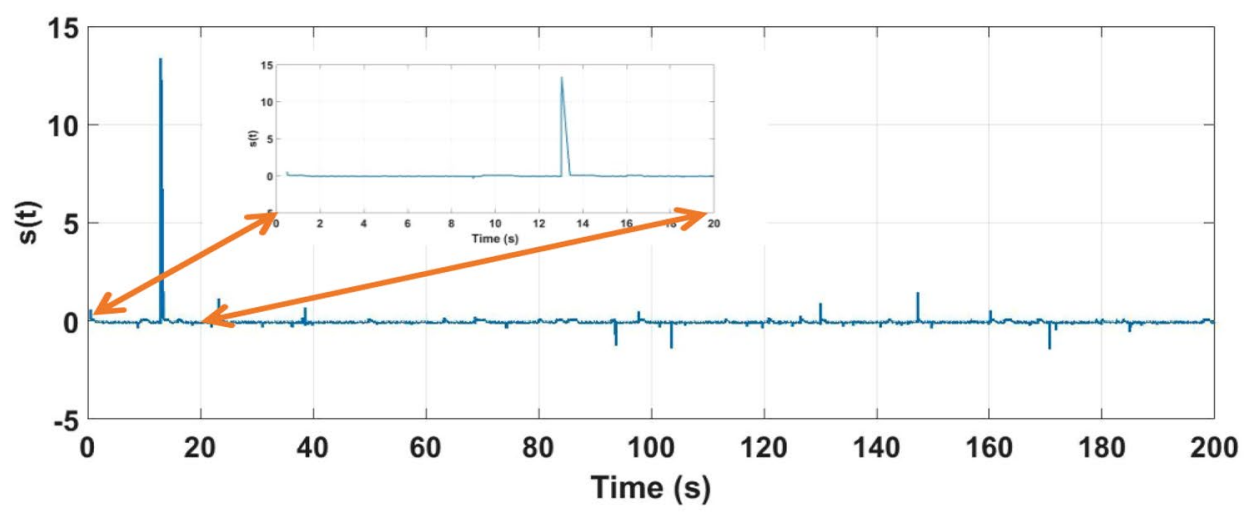

(a)

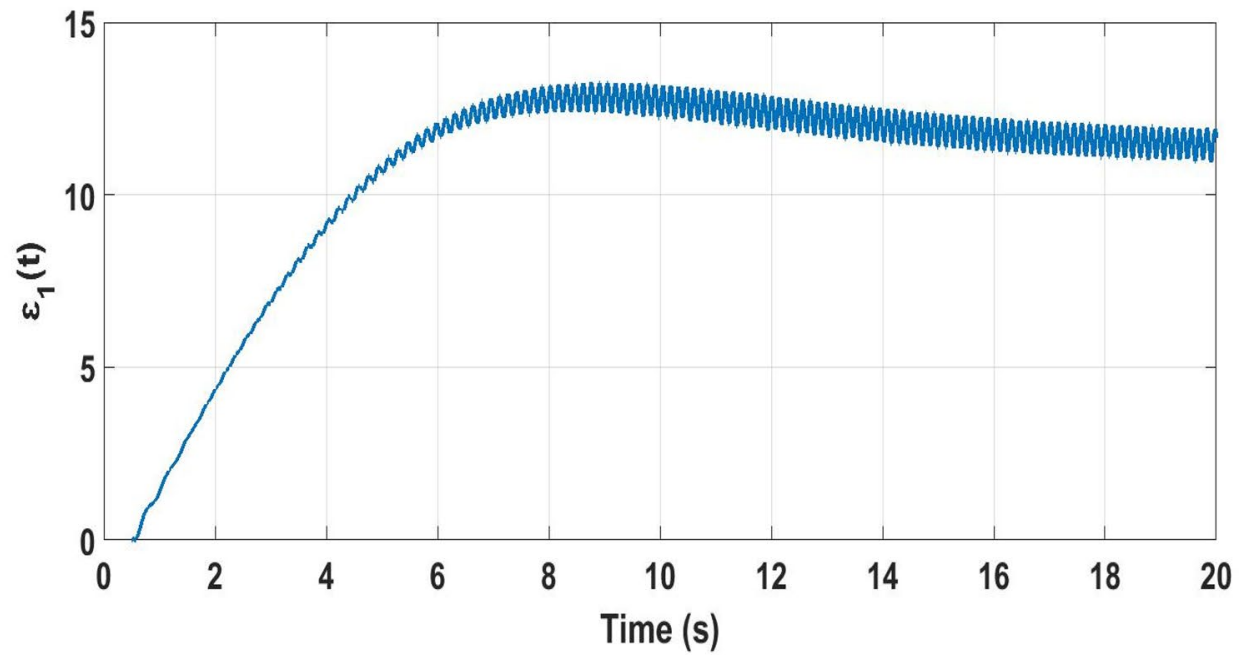

(b)

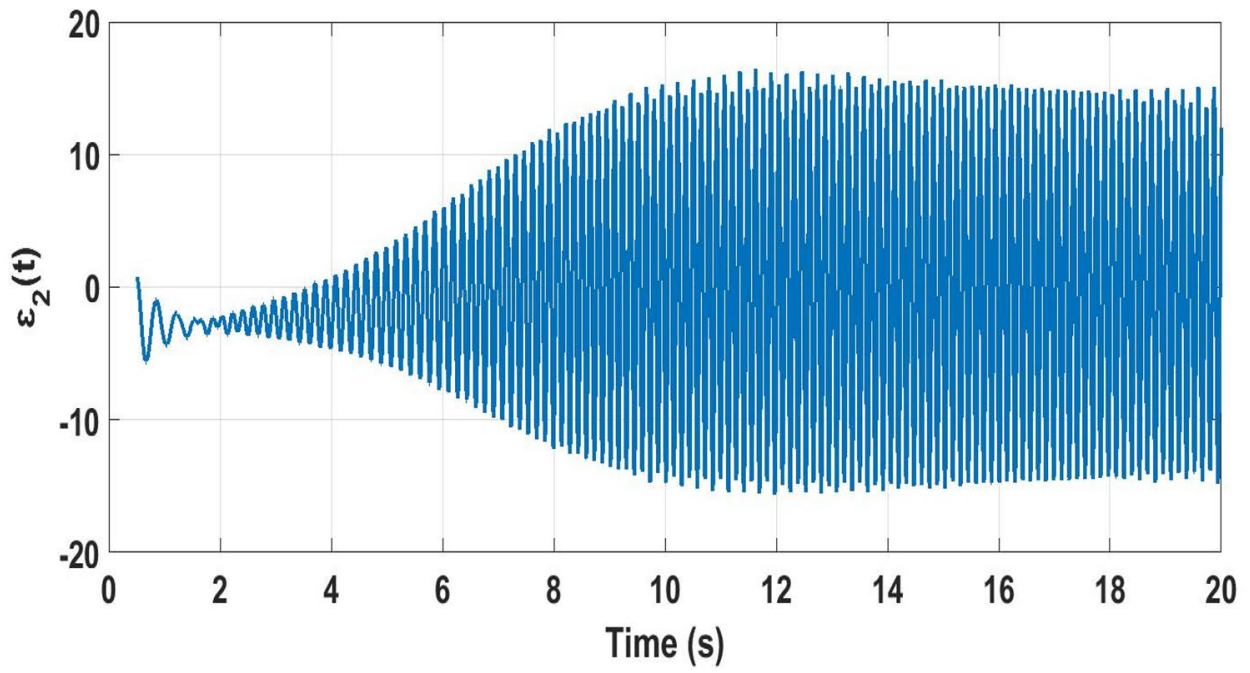

(c) techniques for the Maglev [56, 57]. Although, findings are satisfactory, they remain very conservative and present a real problem of energy consumption. Furthermore, the results obtained in this research are in perfect agreement with the theoretical results and provide a significant contribution to the Maglev control domain. 
Fig. 9 Evolution of the errors of the maglev controlled system

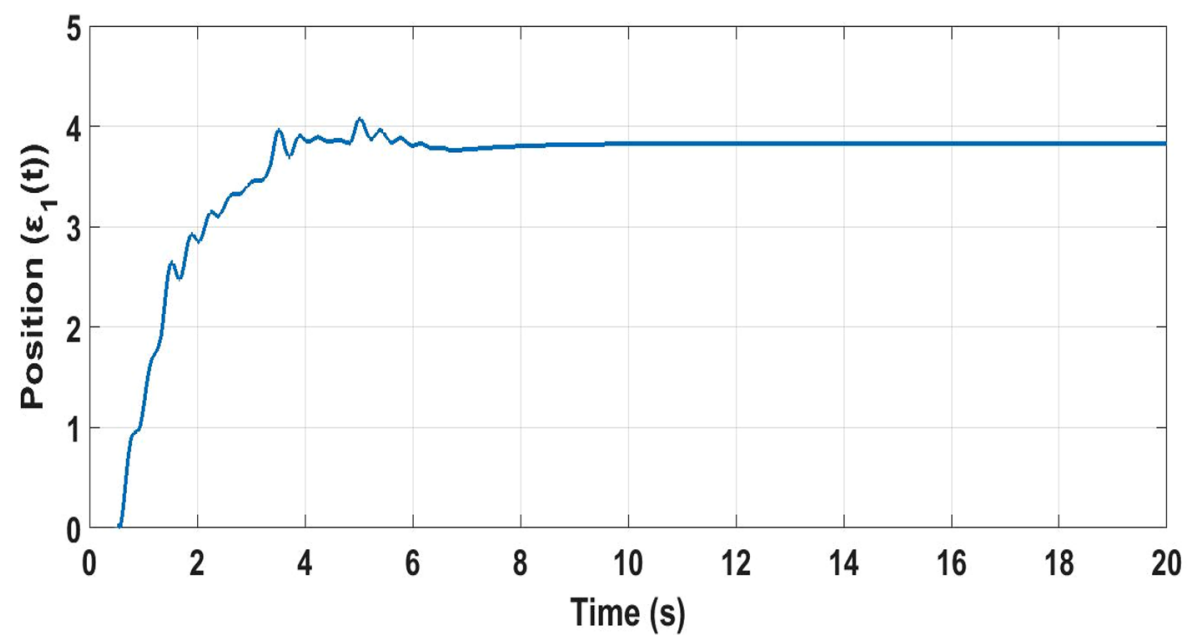

(a)

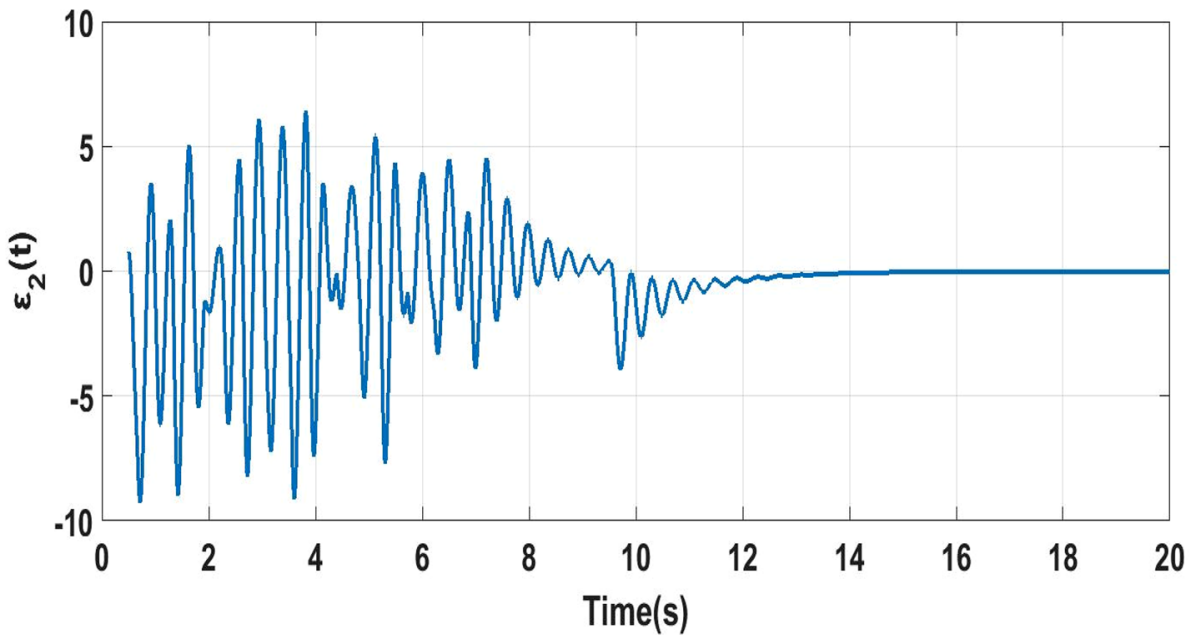

(b)

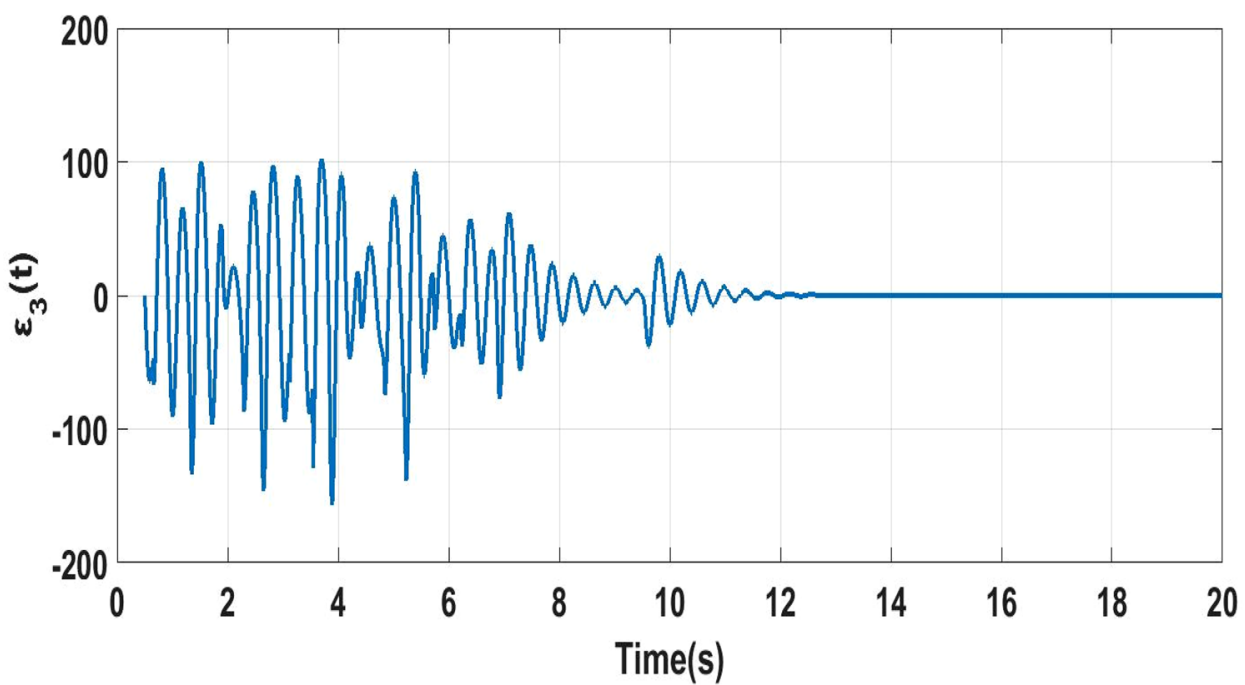

(c) 
Fig. 10 Sliding controller surface $s(t)$

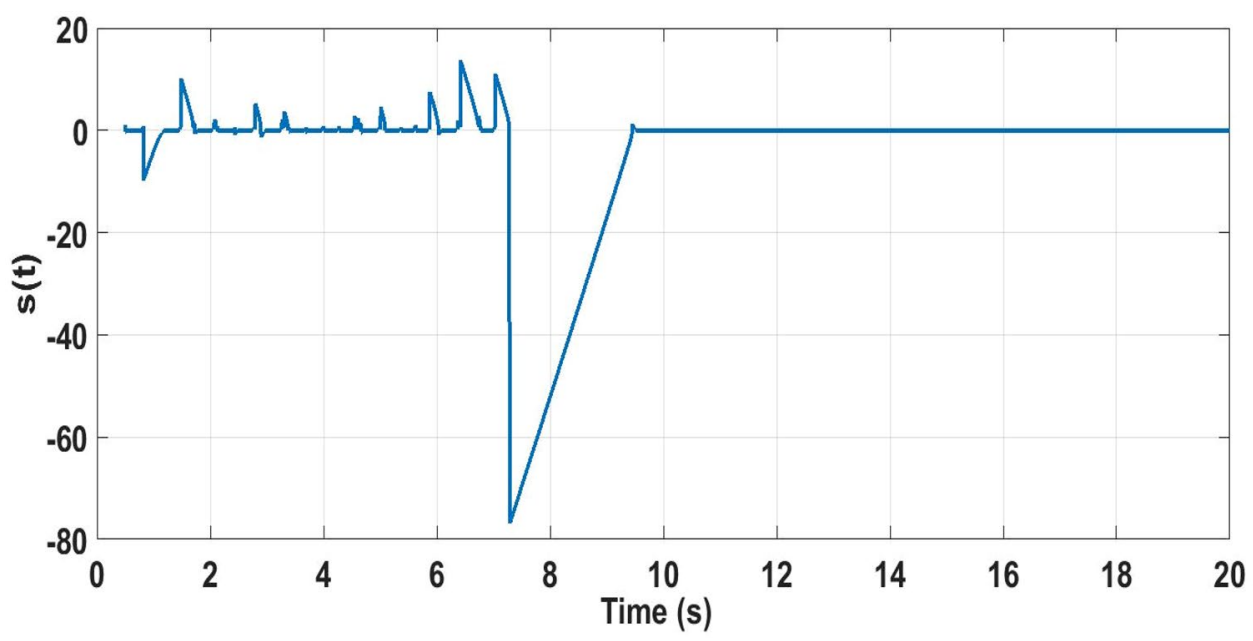

Fig.11 Feedback control gain $k(t)$

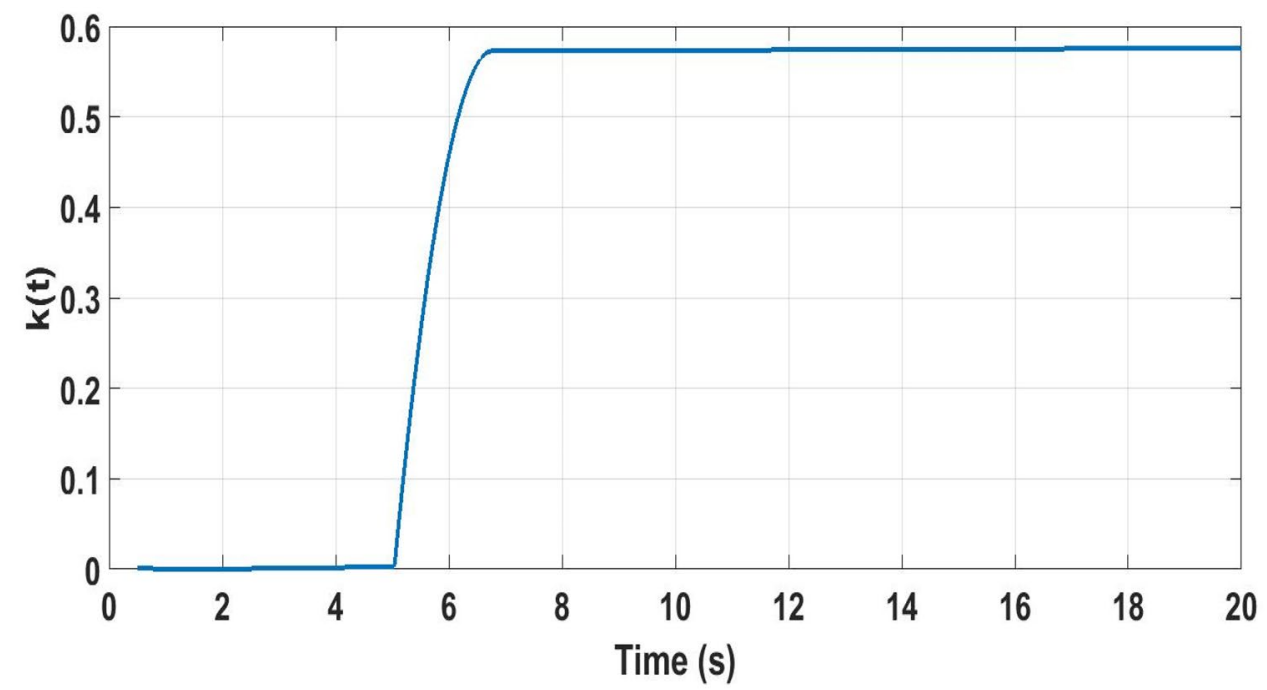

\section{Conclusion}

This work explores a new and simple concept of the critical analysis for the motion stability of a general class of Maglev train incorporated into differential equations. This paper discussed the AC equilibrium point and its stability. It is found that the initial value of the displacement varies between unstable and stable intervals with the amplitude of the control input. To investigate numerically the complex Maglev dynamic, the derivation of the equilibrium point and the corresponding stability analysis is performed and the behavior depending on the control input have revealed the behavior of the system's states with respect to the vibrating frequencies. The analytical studies and numerical ones show perfect agreement and can allow predicting efficiently the displacement's amplitude of the electromagnet as well as the current flowing in the coil. These features are of great interest in the design of maglev vehicles. The adaptive robust nonlinear sliding mode control for the maglev system is proposed in the second part of this work. The bound of uncertainty is assumed to be known. To deal with the difficulties face in Refs. [38], the robust nonsingular sliding mode controller is designed in the frequency domain which guarantees the uniform boundedness of the system regardless of the disturbances. From this study, we can point out a major drawback such as an accurate analytical expression of the displacement and the current due to the high nonlinearity found in the Maglev system.

Our next investigation will be on the test of the present model when the edge effect and the magnetic flux leakage are not neglected, consequently, the flux is not uniformly distributed in the air gap. We will propose to use the fuzzy logic issue to reduce the complexities in analysis and control design. We think also that the analysis of the proposed system with fractional damping is another good idea. 


\section{Compliance with ethical standards}

Conflict of interest The authors declare that there are no conflicts of interest, regarding this paper.

Open Access This article is licensed under a Creative Commons Attribution 4.0 International License, which permits use, sharing, adaptation, distribution and reproduction in any medium or format, as long as you give appropriate credit to the original author(s) and the source, provide a link to the Creative Commons licence, and indicate if changes were made. The images or other third party material in this article are included in the article's Creative Commons licence, unless indicated otherwise in a credit line to the material. If material is not included in the article's Creative Commons licence and your intended use is not permitted by statutory regulation or exceeds the permitted use, you will need to obtain permission directly from the copyright holder. To view a copy of this licence, visit http://creativecommons .org/licenses/by/4.0/.

\section{Appendix}

Resolution of the Eq. (12) using Cardan method

$f\left(y_{0}\right)=K R^{2} y_{0}^{3}-R^{2}\left(K y_{e}+m g\right) y_{0}^{2}+k_{0} u^{2}(t)=0$

Let us set $y_{0}=X+a$ with $a=\frac{K y_{e}+m g}{3 K}$ leading to

$f(X)=K R^{2} X^{3}-\frac{\left(K y_{e}+m g\right)^{2}}{3 K} R^{2} X-\frac{2 R^{2}\left(K y_{e}+m g\right)^{3}}{27 K^{2}}+k_{0} u^{2}(t)=0$

Let us now find $X$ as a linear combination of two independent variables $X_{1}$ and $X_{2}$ such as $X=X_{1}+X_{2}$, leading to

$$
\begin{aligned}
& K R^{2}\left(X_{1}^{3}+X_{2}^{3}\right)+R^{2}\left(X_{1}+X_{2}\right) \frac{9 K^{2} X_{1} X_{2}-\left(K y_{e}+m g\right)^{2}}{3 K} \\
& -2 R^{2} \frac{\left(K y_{e}+m g\right)^{3}}{27 K^{2}}+k_{0} u^{2}(t)=0
\end{aligned}
$$

which can be rewritten as the following set of equations:

$\left\{\begin{array}{l}X_{1}^{3}+X_{2}^{3}=\frac{2\left(K y_{e}+m g\right)^{3}}{27 K^{2}}-\frac{k_{0} u^{2}(t)}{K R^{2}} \\ X_{1}^{3} X_{2}^{3}=\left(\frac{K y_{e}+m g}{3 K}\right)^{6}\end{array}\right.$

which can lead to the following equation

$$
Z^{2}-\left(\frac{2\left(K y_{e}+m g\right)^{3}}{27 K^{2}}-\frac{k_{0} u^{2}(t)}{K R^{2}}\right) Z+\left(\frac{K y_{e}+m g}{3 K}\right)^{6}=0
$$

The discriminant of this equation can be read:

$\delta=\frac{k_{0} u^{2}(t)}{K^{2} R^{4}}\left(-\frac{4}{27} K k_{0} R^{2}\left(y_{e}+\frac{m g}{K}\right)^{3}+k_{0} u^{2}(t)\right)$

It is obvious that $\delta$ is the same as the expression given by Eq. (18), leading to the following solutions:

- If $\delta>0$, one has $Z_{ \pm}=\frac{1}{2}\left(\left(2 \frac{\left(K y_{e}+m g\right)^{3}}{27 K^{3}}-\frac{k_{0} u^{2}(t)}{K R^{2}}\right) \pm \sqrt{\delta}\right)$, and as a consequence,

$$
\begin{aligned}
& X_{1}^{3}=\frac{1}{2}\left(\left(2 \frac{\left(K y_{e}+m g\right)^{3}}{27 K^{3}}-\frac{k_{0} u^{2}(t)}{K R^{2}}\right)+\sqrt{\delta}\right) \\
& X_{2}^{3}=\frac{1}{2}\left(\left(2 \frac{\left(K y_{e}+m g\right)^{3}}{27 K^{3}}-\frac{k_{0} u^{2}(t)}{K R^{2}}\right)-\sqrt{\delta}\right)
\end{aligned}
$$

$$
\left\{\begin{array}{l}
X_{1}=\left[\frac{1}{2}\left(\left(2 \frac{\left(K_{y}+m g\right)^{3}}{27 K^{3}}-\frac{K_{0} u^{2}(t)}{K R^{2}}\right)+\sqrt{\delta}\right)\right]^{1 / 3} \exp \left(\frac{2 \pi n i}{3}\right), n=0,1,2 \\
X_{2}=\left[\frac{1}{2}\left(\left(2 \frac{\left(K_{\mathrm{e}}+m g\right)^{3}}{27 K^{3}}-\frac{K_{0} u^{2}(t)}{K R^{2}}\right)-\sqrt{\delta}\right)\right]^{1 / 3} \exp \left(-\frac{2 \pi n i}{3}\right), n=0,1,2
\end{array}\right.
$$

Meaning that

$$
\begin{aligned}
& y_{0}=\frac{K y_{e}+m g}{3 K}+\left(\left[\frac{1}{2}\left(\left(2 \frac{\left(K y_{e}+m g\right)^{3}}{27 K^{3}}-\frac{k_{0} u^{2}(t)}{K R^{2}}\right)+\sqrt{\delta}\right)\right]^{1 / 3}+\left[\frac{1}{2}\left(\left(2 \frac{\left(K y_{e}+m g\right)^{3}}{27 K^{3}}-\frac{k_{0} u^{2}(t)}{K R^{2}}\right)-\sqrt{\delta}\right)\right]^{1 / 3}\right) \cos \left(\frac{2 \pi n}{3}\right) \\
& +i\left(\left[\frac{1}{2}\left(\left(2 \frac{\left(K y_{e}+m g\right)^{3}}{27 K^{3}}-\frac{k_{0} u^{2}(t)}{K R^{2}}\right)+\sqrt{\delta}\right)\right]^{1 / 3}-\left[\frac{1}{2}\left(\left(2 \frac{\left(K y_{e}+m g\right)^{3}}{27 K^{3}}-\frac{k_{0} u^{2}(t)}{K R^{2}}\right)-\sqrt{\delta}\right)\right]^{1 / 3}\right) \sin \left(\frac{2 \pi n}{3}\right), n=0,1,2
\end{aligned}
$$

$y_{0}=\frac{K y_{e}+m g}{3 K}+\left(\left[\frac{1}{2}\left(\left(2 \frac{\left(K y_{e}+m g\right)^{3}}{27 K^{3}}-\frac{k_{0} u^{2}(t)}{K R^{2}}\right)+\sqrt{\delta}\right)\right]^{1 / 3}+\left[\frac{1}{2}\left(\left(2 \frac{\left(K y_{e}+m g\right)^{3}}{27 K^{3}}-\frac{k_{0} u^{2}(t)}{K R^{2}}\right)-\sqrt{\delta}\right)\right]^{1 / 3}\right)$

For this particular case, the real solution is the case where $n=0$, that is 
Corresponding to solution (19). The cases $\mathrm{n}=1$ and $n=2$ being complex solutions.

- If $\delta=0$, one has from solution (68)

$$
y_{0}=\frac{K y_{e}+m g}{3 K}+2\left[\frac{1}{2}\left(\left(2 \frac{\left(K y_{e}+m g\right)^{3}}{27 K^{3}}-\frac{k_{0} u^{2}(t)}{K R^{2}}\right)+\sqrt{\delta}\right)\right]^{1 / 3}
$$

$\cos \left(\frac{2 \pi n}{3}\right), n=0,1,2$, leading to solutions:

$\left\{\begin{array}{l}y_{01}=\frac{K y_{e}+m g}{3 K}+2\left[\frac{1}{2}\left(\left(2 \frac{\left(K y_{e}+m g\right)^{3}}{27 K^{3}}-\frac{k_{0} u^{2}(t)}{K R^{2}}\right)\right)\right]^{1 / 3} \\ y_{02}=\frac{K y_{e}+m g}{3 K}-\left[\frac{1}{2}\left(\left(2 \frac{\left(K y_{e}+m g\right)^{3}}{27 K^{3}}-\frac{k_{0} u^{2}(t)}{K R^{2}}\right)\right)\right]^{1 / 3}\end{array}\right.$

\section{Corresponding to solution (20).}

If $\delta<0$; one has from A7;

$$
\begin{aligned}
& Z_{ \pm}=\frac{1}{2}\left(\left(2 \frac{\left(K y_{e}+m g\right)^{3}}{27 K^{3}}-\frac{k_{0} u^{2}(t)}{K R^{2}}\right) \pm i \sqrt{-\delta}\right)=r \exp ( \pm i \theta), \\
& \text { with } r^{2}=2 \frac{\left(K y_{e}+m g\right)^{3}}{27 K^{3}}-\frac{k_{0} u^{2}(t)}{K R^{2}}-\delta=\frac{4}{729}\left(y_{e}+\frac{m g}{K}\right)^{6} \text {, and } \\
& \tan (\theta)=\frac{\sqrt{-\delta}}{2 \frac{\left(K y_{e}+m g\right)^{3}}{27 K^{3}}-\frac{k_{0} u^{2}(t)}{K R^{2}}}
\end{aligned}
$$

Leading to

$$
\left\{\begin{array}{c}
X_{1}=\frac{1}{3}\left(y_{e}+\frac{m g}{K}\right) \exp \left(i \frac{\theta+2 \pi n}{3}\right) \\
X_{2}=\frac{1}{3}\left(y_{e}+\frac{m g}{K}\right) \exp \left(-i \frac{\theta+2 \pi n}{3}\right)
\end{array} \quad n=0,1,2\right.
$$

Finally, we have

$$
y_{0}=\frac{K y_{e}+m g}{3 K}+\frac{1}{3}\left(y_{e}+\frac{m g}{K}\right) \cos \left(\frac{\theta+2 \pi n}{3}\right), n=0,1,2,
$$

which is the solution given by (21).

\section{References}

1. Xiaoming $Z$, Wanming $L$ (2016) Key technologies in the construction of medium and low speed maglev in Changsha City. Urban Mass Transit 19:1-4

2. Sun Y, Xu J, Qiang H, Lin G (2019) Adaptive neural-fuzzy robust position control scheme for maglev train systems with experimental verification. IEEE Trans Ind Electron 66(11):8589-8599

3. Chen C, Xu J, Ji W, Rong L, Lin G (2019) Sliding mode robust adaptive control of maglev Vehicle's nonlinear suspension system based on flexible track: Design and experiment. IEEE Access 7:41874-41884

4. Tezuka N, Kurita T, Ishikawa T (2013) Design and simulation of a five degrees of freedom active control T magnetic levitated motor,". IEEE Trans Magn 49(5):2257-2262
5. Wang H, Li F (2018) Levitation control of an improved modular bearingless switched reluctance motor. ISA Trans 80:564-571

6. Osa, M., Masuzawa, T., Orihara, R., and Tatsumi, E., (2017). Compact maglev motor with full DOF active control for miniaturized rotary blood pumps," in Proc. 11th Int. Symp. Linear Drives Ind. Appl. (LDIA), Osaka, Japan, 1-6.

7. Kim C (2019) Robust air-gap control of superconductinghybrid MagLev intelligent conveyor system in smart factory. IEEE Trans Magn 55(6):8300705

8. Verma S, Kim W, Shakir H (2005) Multi-axis maglev nanopositioner for precision manufacturing and manipulation applications. IEEE Trans Ind Appl 41(5):1159-1167

9. Cao R, Jin Y, Lu M, Zhang Z (2018) Quantitative comparison of linear flux-switching permanent magnet motor with linear induction motor for electromagnetic launch system. IEEE Trans Ind Electron 65(9):7569-7578

10. Hull JR, Fiske J, Ricci K, Ricci M (2007) Analysis of levitational systems for a superconducting launch ring".. IEEE Trans Appl Supercond 17(2):2117-2120

11. Lin FJ, Teng LT, Shieh PH (2007) Intelligent adaptive backstepping control system for magnetic levitation apparatus. IEEE Transac Magnetic 43(5):2009-2018

12. Zhou $H$, Ying $H$, Duan J (2011) Adaptive control using interval Type-2 fuzzy logic for uncertain nonlinear systems. J Central South Univ Technol 18(3):760-766

13. Yougang S, Wanli L, Junqi X, Haiyan Q, Chen C (2017) Nonlinear dynamic modeling and fuzzy sliding-mode controlling of electromagnetic levitation system of low-speed maglev train. JVE international Itd journal of vibroengineering 19:1392-8716

14. Lee JS, Kwon SD, Kim MY et al (2009) A parametric study on the dynamics of urban transit Maglev vehicle running on flexible guideway bridges. J Sound Vib 32:301-317

15. Haiyan Q, Wanli L, Yougang S, Xiangyong L (2017) A levitation chassis dynamic analysis and robust position control for maglev vehicles under nonlinear periodic disturbance. JVE Int Itd J Vibroeng 19:1392-8716

16. Mingda $Z$, Zhiqiang $L$, Xiaolong $L$ (2019) Calculation and evaluation of load performance of magnetic levitation system in medium-low speed maglev train. Int J Appl Electromag Mech $1: 1-18$

17. You-gang S, Si X, Jun-qi X, Guo-bin L (2020) A robust levitation control of maglev vehiclessubject to time delay and disturbances: designand hardware experimentation. Appl Sci 10:1179. https://doi.org/10.3390/app10031179

18. Khan $\mathrm{M}$ et al (2016) Robust $\mathrm{H}_{1}$ control of Magnetic Levitation system based on parallel distributed compensator. Ain Shams Eng J. https://doi.org/10.1016/j.asej.2016.06.005

19. Osa, M., Masuzawa, T., Orihara, R., and Tatsumi, E., (2017). Compact maglev motor with full DOF active control for miniaturized rotary blood pumps. in Proc. 11 th Int. Symp. Linear Drives Ind. Appl. (LDIA), Osaka, Japan, 1-6.

20. Barie W, Chiasson J (1996) Linear and nonlinear state-space controllers for magnetic levitation. Int J Syst Sci 27(11):1153-1163

21. Trumper DL, Olson SM, Subrahmanyan PK (1997) Linear zing control of magnetic suspension systems. Control Syst Technol IEEE Transact 5(4):427-438

22. Wang $H$, Zhong $X$, Shen $G$ (2015) Analysis and experimental study on the MAGLEV vehicle-guideway interaction based on the full-state feedback theory. J Vib Control 21:408-416

23. Malik AS, Ahmad A, Rahman U, Islam Y (2019) Integral backstepping and synergetic control of magnetic levitation system. IEEE Access 7:173230-173239

24. Yang Z, Miyazaki K, Kanae S et al (2004) Robust position control of a magnetic levitation system via dynamic surface control technique. IEEE Trans Ind Electron 51:26-34 
25. Lee JD, Khoo S, Wang ZB (2013) DSP-based sliding-mode control for electromagnetic-levitation precise-position system. IEEE Trans Ind Inf 9(2):817-827

26. Wiboonjaroen W, Sujitjorn S (2012) State-PID feedback for magnetic levitation system. Adv Mater Res 622(623):1467-1473

27. Bobtsov AA, Pyrkin AA, Ortega RS, Vedyakov AA (2018) A state observer for sensorless control of magnetic levitation systems. Automatica 97:263-270

28. Sun Y, Xu J, Lin G, Ji W, Wang L (2020) RBF Neural Network-Based Supervisor Control for Maglev Vehicles on an Elastic Track with Network Time-Delay,." IEEE Transact Indus Info in press,. https:// doi.org/10.1109/TII.2020.3032235

29. Junqi X, Yuan Z (2011) A nonlinear control method for the electromagnetic suspension system of the maglev train. J Modern Transport 19:176-180

30. Junqi X, Chen C, Dinggang G, Shihui L, Qingquan Q (2017) Nonlinear dynamic analysis on maglev train system with flexible guideway and double time-delay feedback control. JVE Int Itd J Vibroeng 19:1392-8716

31. Sun Y, Li W, Xu J et al (2017) Nonlinear dynamic modeling and fuzzy sliding-mode controlling of electromagnetic levitation system of low-speed maglev train. J Vibroeng 19:328-342

32. Zhang M, Gao C, Ma WH (2018) Effect of different connection modes of electromagnets on the performance of levitation control. Proc Inst Mech Eng Part F: J Rail Rapid Transit 232:2111-2125

33. Utkin VI (1977) Sliding mode systems with sliding modes. IEEE Transact Auto Control 22:212-222

34. Huang CF, Lin JS, Liao TL, Yan JJ (2011) Quasi sliding mode control for chaotic symmetric gyros with linear-plus-cubic damping and input nonlinearity. J Vibr Control 18:1330-1335

35. Ming-Chang P (2019) Synchronization of unified chaotic systems via adaptive nonsingular fast terminal sliding mode control. Int J Dyn Control. https://doi.org/10.1007/s40435-018-0486-z

36. Draženovic B (1969) The invariance conditions in variable structure systems. Automatica 5:287-295

37. Yao Z, Bin X, Shugen M (2015) Continuous robust tracking control for magnetic levitation system with unidirectional input constraint. IEEE Transact Ind Electron 62:5971-5980

38. Kammogne ST, Ahmad TA, Kengne R, Fotsin HB (2019) Robust observer-based synchronization of chaotic oscillators with structural perturbations and input nonlinearity. Int J Auto Control 13:387-412

39. Nirmal P, Jonathan Z (2013) Modeling the dynamic electromechanical suspension behavior of an electrodynamic eddy current maglev device. Progress Electromag Res 49:1-30

40. Zhang M, MA W, and Luo S (2019). Application of levitation frame with mid-set air spring on maglev vehicles. IOP Conf. Series: Journal of Physics: Conf. Series 1187032035 IOP Publishing doi:https://doi.org/10.1088/1742-6596/1187/3/032035

41. Zhang $H$, Kou $B$, Jin $Y$, Zhang $H$, Zhang $L$ (2014) Research on a Low Stiffness Passive Magnetic Levitation Gravity Compensation System with Opposite Stiffness Cancellation. IEEE Transact Magn 50(11):1-4

42. Yuan NH, Yan L, (2015). Stability design of maglev actuator in active vibration control. International Conference on Information Sciences, Machinery, Materials and Energy (ICISMME 2015).
43. Jinquan Xu, Ye-Hwa C, Hong G (2015) Robust levitation control for maglev systems with guaranteed bounded airgap. ISA Transact 59:205-214

44. Yamada $T$, Iwamoto $M$, Ito $T$ (1974) Magnetic damping force in inductive magnetic levitation system for high-speed trains. Elect Eng Japan 94(1):49-54

45. Seok-Kyoon K (2019) Nonlinear position stabilizing control with active damping injection technique for magnetic levitation systems. Electron 8:221. https://doi.org/10.3390/electronics8020 221

46. Shi J, Wei Q, Zhao Y (2007) Analysis of dynamic response of the high-speed EMS maglev vehicle/guideway coupling system with random irregularity. Veh Syst Dyn. 45:1077-1095

47. Wang Keren, Luo Shihui, Ma Weihua, Chen Xiaohao (2017) Dynamic characteristics analysis for a new-type maglev vehicle. Adv Mech Eng. https://doi.org/10.1177/1687814017745415

48. Talukdar RP, Talukdar S (2016) Dynamic analysis of high-speed MAGLEV vehicle-guideway system: an approach in block diagram environment. Urban Rail Transit 2(2):71-84. https://doi. org/10.1007/s40864-016-0039-8

49. Sinha, P.K. (1987), Electromagnetic suspension-dynamics and control, IEEE.

50. Tsunashima $H, A$ be $M(1998)$ Static and dynamic performance of permanent magnet suspension for maglev transport vehicle. Vehicle Syst Dyn 29(2):83-111

51. Xu J, Zhou Y (2011) A nonlinear control method for the electromagnetic suspension system of the maglev train. J Modern Transp 19(3):176-180

52. Hideki K (2017) History of air spring development for shinkansen trains. SEI Tech Rev 84:114-119

53. Geng Z, Jie L, (2011). Dynamical model and characteristics analysis for air spring system of low-speed Maglev train. International conference on Advanced Computer Theory and Engineering, $4^{\text {th }}$ (ICACTE 2011). Ed. Yi Xie. ASME Press, 2011

54. Han HS, Moon SJ, Cho HW (2003) A study on the dynamic modeling of a magnetic levitation vehicle. JSME Int J Ser C 46(4):1497-1501

55. Bocheng B, Pan J, Huagan W, Fengwei H (2014) Complex transient dynamics in periodically forced memristive Chua's circuit. Nonlinear Dyn 79:2333-2343. https://doi.org/10.1007/s1107 1-014-1815-1

56. Wouapi M, Fotsin H, Louodop P, Feudjio F, Njitacke T, Djeudjo H (2020) Various firing activities and finite-time sysnchronization of an improved Hindmarsh-rose neuron model under electric field effect. Cognit Neurodyn. https://doi.org/10.1007/s1157 1-020-09570-0

57. Guang H, Jie L, Peng C (2016) Nonlinear control scheme for the levitation module of maglev train. J Dyn Sys Meas Control 138:074503

58. Won-jong K, Shobhit V (2007) Multi axis maglev positioner with nanometer resolution over extended travel range. J Dyn Syst Meas Control 129:777-785

Publisher's Note Springer Nature remains neutral with regard to jurisdictional claims in published maps and institutional affiliations. 